\title{
Using VO tools to investigate distant radio starbursts hosting obscured AGN in the $\operatorname{HDF}(\mathrm{N})$ region ${ }^{\star}$
}

\author{
A. M. S. Richards ${ }^{1}$, T. W. B. Muxlow ${ }^{1}$, R. Beswick ${ }^{1}$, M. G. Allen ${ }^{2}$, K. Benson ${ }^{3}$, R. C. Dickson ${ }^{1}$, M. A. Garrett ${ }^{4}$, \\ S. T. Garrington ${ }^{1}$, E. Gonzalez-Solarez ${ }^{5}$, P. A. Harrison ${ }^{6}$, A. J. Holloway ${ }^{1}$, M. M. Kettenis ${ }^{7}$, R. A. Laing ${ }^{6}$, \\ E. A. Richards ${ }^{8}$, H. Thrall ${ }^{1}$, H. J. van Langevelde ${ }^{7,9}$, N. A. Walton ${ }^{5}$, P. N. Wilkinson ${ }^{1}$, and N. Winstanley ${ }^{1}$ \\ 1 Jodrell Bank Observatory, University of Manchester, SK11 9DL, Macclesfield, UK \\ e-mail: amsr@jb.man.ac.uk \\ 2 Centre de Données astronomiques de Strasbourg (UMR 7550), 67000 Strasbourg, France \\ Mullard Space Science Laboratory, UCL, Holmbury St. Mary, Dorking, Surrey, RH5 6NT, UK \\ 4 Netherlands Foundation for Research in Astronomy, Postbus 2, 7990 AA Dwingeloo, The Netherlands \\ Institute of Astronomy, Madingley Road, Cambridge, CB3 0HA, UK \\ ${ }^{6}$ European Southern Observatory, 85748 Garching bei München, Germany \\ Joint Institute for VLBI in Europe, Postbus 2, 7990 AA Dwingeloo, The Netherlands \\ 8 Department of Physics, Talledega College, Talledega, Alabama 35160, USA \\ 9 Sterrewacht Leiden, Leiden University, Postbus 9513, 2300 RA Leiden, The Netherlands
}

Received 3 April 2007 / Accepted 21 June 2007

\section{ABSTRACT}

Context. A 10-arcmin region around the Hubble Deep Field (North) contains 92 radio sources brighter than $40 \mu \mathrm{Jy}$ which are wellresolved by MERLIN+VLA at $0.2-2^{\prime \prime}$ resolution (average size $\sim 1^{\prime \prime}$ ). 55 of these have Chandra X-ray counterparts in the 2-Ms $\mathrm{CDF}(\mathrm{N})$ field including at least 17 with a hard X-ray photon index and high luminosity characteristic of a type-II (obscured) AGN. More than $70 \%$ of the radio sources have been classified as starbursts or AGN using radio morphologies, spectral indices and comparisons with optical appearance and rest-frame MIR emission. On this basis, starbursts outnumber radio AGN 3:1.

Aims. We investigate the possibility that very luminous radio and X-ray emission originates from different phenomena in the same high-redshift galaxies.

Methods. This study extends the Virtual Observatory (VO) methods previously used to identify X-ray-selected obscured type-II AGN, to examine the relationship between radio and X-ray emission. We describe a VO cut-out server for MERLIN+VLA 1.4-GHz radio images in the $\mathrm{HDF}(\mathrm{N})$ region.

Results. The high-redshift starbursts have typical sizes of 5-10 kpc and star formation rates of $\sim 1000 M_{\odot} \mathrm{yr}^{-1}$, an order of magnitude more extended and intense than in the local universe. There is no obvious correlation between radio and X-ray luminosities nor spectral indices at $z \gtrsim 1.3$. About $70 \%$ of both the radio-selected AGN and the starburst samples were detected by Chandra. The X-ray luminosity indicates the presence of an AGN in at least half of the 45 cross-matched radio starbursts. Eleven of these are type-II AGN, of which 7 are at $z \geq 1.5$. This distribution overlaps closely with the X-ray detected radio sources which were also detected by SCUBA. In contrast, all but one of the AGN-dominated radio sources are at $z<1.5$, including the 4 which are also X-ray selected type-II AGN. The stacked 1.4-GHz emission at the positions of radio-faint X-ray sources is correlated with X-ray hardness. Conclusions. Almost all extended radio starbursts at $z>1.3$ host X-ray selected obscured AGN. The radio emission from most of these ultra-luminous objects is dominated by star formation although the highest redshift $(z=4.424)$ source has a substantial AGN contribution. Star-formation appears to contribute less than $1 / 3$ of their X-ray luminosity. Our results support the inferences from SCUBA and IR data, that at $z \gtrsim 1.5$, star formation is observably more extended and more copious, it is closely linked to AGN activity and it is triggered differently, compared with star formation at lower redshifts.

Key words. astronomical data bases: miscellaneous - X-rays: galaxies - radio continuum: galaxies - galaxies: active galaxies: starburst - galaxies: evolution

\section{Introduction}

There is now general agreement that the number of vigorous starforming galaxies, and the star formation rate (SFR) within these galaxies, increases dramatically at $z>1$. The details of how these starburst galaxies relate to the high redshift Active Galactic Nucleus (AGN) population are less clear. Objects detected individually at $z>1$ in radio and X-rays, by even the deepest

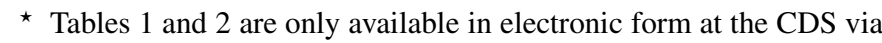
anonymous ftp to cdsarc.u-strasbg.fr $(130.79 .128 .5)$ or via http://cdsweb.u-strasbg.fr/cgi-bin/qcat?]/A+A/472/805 available exposures, are inevitably abnormally luminous. Is it equally inevitable that, in a galaxy detected in both regimes, all such bright emission emanates from the same phenomenon, or can we separate contributions from AGN and from starbursts if these coexist?

The unprecedentedly sensitive observations of the Hubble Deep Field (North) $\left(\mathrm{HDF}(\mathrm{N})^{1}\right)$ which commenced in 1996

1 We use the term $\operatorname{HDF}(\mathrm{N})$ to describe observations made in and around the original HDF; these form part of the multiwavelength GOODS (Great Observatories Origins Deep Surveys) project (Giavalisco et al. 2004). 
provided the first detailed attempts to quantify the star formation history of the universe (Madau et al. 1996). The radio luminosity function evolves rapidly with redshift, as $(1+z)^{3}$ for $0.5<z<1.5$ (Cowie et al. 2004a). Subsamples classified using optical spectra and X-ray power suggest that the AGN luminosity function is declining at $z>0.9$ compared to lower redshifts, while the reverse is the case for star-forming galaxies. This is supported by Spitzer detections of Ultra-Luminous IR Galaxies (ULIRGs) with IR luminosities $>10^{12} M_{\odot}$. These show that the co-moving density of ULIRGs (with a typical SFR of 200-300 $M_{\odot} \mathrm{yr}^{-1}$ ) at $z \sim 2$ was at least 3 orders of magnitude greater than in the local universe (Daddi et al. 2005).

Star-formation rates (SFR) measured from optical data only can be greatly underestimated or overlooked altogether (Reddy $\&$ Steidel 2004). For example, Cowie et al. (2004a), using optical spectra, were only able to classify $53 \%$ of the radio sources $<100 \mu \mathrm{Jy}$ in the $\operatorname{HDF}(\mathrm{N})$, finding that $28 \%$ are star forming galaxies. In contrast, over $2 / 3$ of the 58 resolved sources $<100 \mu \mathrm{Jy}$ in the $\mathrm{HDF}(\mathrm{N})$ were classified using the radio-based criteria of Muxlow et al. (2005), containing 60\% starbursts. Similarly, up to $90 \%$ of the distant or obscured AGN revealed by deep X-ray observations may be missed by optical surveys (Bauer et al. 2004).

Classification based on IR, sub-mm and radio properties is favoured because local starburst galaxies show a strong peak in their spectral energy distributions (SED) around $3 \mathrm{THz}(100 \mu \mathrm{m})$ (e.g. Yun \& Carilli 2002) which can be used to estimate the SFR (Condon 1992; Cram et al. 1998; Yun et al. 2001). The most striking evidence for extraordinary levels of high-redshift star formation came from Sub-mm Common User Bolometer Array (SCUBA) observations (Hughes et al. 1998; Smail et al. 2002; review by Blain et al. 2002). The median redshift for SCUBA sources (SMG) in the HDF(N) with optical counterparts is at least 2. SMG have a typical SFR of 1000-2000 $M_{\odot} \mathrm{yr}^{-1}$, an order of magnitude greater than in the most active local ULIRGs such as Arp 220 (SFR 50-150 $M_{\odot} \mathrm{yr}^{-1}$ ). The FIR intensity is well-correlated with radio emission (on scales greater than a few tens of pc) (Condon 1992; Yun et al. 2001). Elbaz et al. (2002), Garrett (2002) and Chapman et al. (2005) have shown that the relationship is valid out to at least $z \sim 3$.

Star formation dominates the rest-frame MIR and FIR output even if an AGN is present (Downes \& Solomon 1998; Frayer et al. 1998) as emission due to dust heating by AGN declines steeply from the NIR to the FIR (e.g. Markarian 231, Soifer et al. 2000). The observed ratio of $X$-ray to rest frame FIR luminosity is $\$ 10 \%$ in local active galaxies even when a strong AGN is present and lower still at high redshifts, especially for starburstdominated sources (Alexander et al. 2003a, 2005a).

Almost half of the optical spectra available for 2-Ms X-ray sources in the $\operatorname{HDF}(\mathrm{N})$ indicate the presence of star formation in the same galaxy (Sadler et al. 2002; Barger et al. 2005). There is evidence that radio and X-ray emission has a common origin in starforming galaxies at relatively low redshifts (Alexander et al. 2002). Bauer et al. (2002b) derive a relationship between radio and X-ray luminosities for 102 emission-line galaxies at $z \leq 1.3$ (of which only 2 sources at $z>1$ were detected in both radio and $\mathrm{X}$-rays in the data then available):

$\log L_{\mathrm{X}}=(0.935 \pm 0.073) \log L_{\mathrm{R}}+(13.141 \pm 1.650)$

where $L_{\mathrm{X}}$ and $L_{\mathrm{R}}$ are the X-ray and radio rest-frame luminosities in $\mathrm{W}$ and $\mathrm{W} \mathrm{Hz}{ }^{-1}$. This does not seem to hold so well for samples extending to higher redshift; Barger et al. (2007) find no correlation between radio and X-ray luminosities for optically-classified star-forming galaxies in the $\operatorname{HDF}(\mathrm{N})$ brighter than $60 \mu \mathrm{Jy}$.

In this paper, we investigate whether the relationship holds at high redshift using classifications independent of optical detections and we explore the properties of radio counterparts to the obscured AGN (type-II AGN) identified from their hard X-ray photon indices and high X-ray luminosities by Padovani et al. (2004).

Many investigations of high-redshift star formation deliberately exclude AGN hosts. We do not need to do this because we use sub-arcsec resolution to distinguish between different energy sources in the same galaxy, which may correspond to different classifications in different wavelength regimes. The whole field has only been well-resolved by the HST and by MERLIN+VLA at $1.4 \mathrm{GHz}$. The extent of radio emission from high-redshift galaxies in the $\operatorname{HDF}(\mathrm{N})$ is typically $1^{\prime \prime}-2^{\prime \prime}$.

We present the first detailed comparision between the highest sensitivity MERLIN+VLA and Chandra data ever taken and the HST ACS images. The data used in this paper are described in more detail in Sect. 2, followed by a summary of the Virtual Observatory and RadioNet ${ }^{2}$ software which has made these results possible, in Sect. 3.1. In Sects. 4 and 5 we explain how we derive the radio and X-ray luminosities ${ }^{3}$ and deduce the origins of the emission, based primarily radio data for the radio sources and X-ray data for X-ray sources. Their relationships are explored in Sect. 6 and we present evidence for the presence of embedded type-II AGN in radio starbursts in Sect. 7. We demonstrate statistically the presence of faint radio emission associated with the majority of X-ray sources in Sect. 8 and summarise our conclusions in Sect. 9.

\section{2. $\operatorname{HDF}(\mathrm{N})$ data, cross-identifications and redshifts}

In this section we introduce the radio observations and describe briefly the X-ray and other data and tools used to make comparisons. The positions, flux densities and spectral and photon indices of radio sources with X-ray counterparts are listed in Table 1, along with their redshifts and any IR or sub-mm detections. All positions given in this paper have been aligned with the VLA or MERLIN+VLA data as these provide the most accurate reference frame, aligned with the International Celestial Reference Frame (ICRF) to better than 15 milli-arcsec (mas) (Muxlow et al. 2005).

\subsection{Radio observations of the $\operatorname{HDF}(N)$}

Muxlow et al. (2005), Richards (2000) and Richards et al. (1998) describe the MERLIN and VLA observations of the HDF(N) made in 1996-7. The VLA-only 1.4-GHz image contains 92 sources above its completeness limit of $40 \mu \mathrm{Jy}$ per $2^{\prime \prime}$ beam $(\sim 5.5 \sigma)$ in a box of side $10^{\prime}$ (the 10 -arcmin field), the "radio-bright" sample. The MERLIN field was centred on Right Ascension $12^{\mathrm{h}} 36^{\mathrm{m}} 49^{\mathrm{s}} .4000$, Declination $+62^{\circ} 12^{\prime} 58^{\prime \prime} .000$ (J2000), hereafter taken as the reference position.

\subsection{GHz MERLIN+VLA observations and other radio data}

The combined MERLIN+VLA $1.4 \mathrm{GHz}$ data reach an rms noise level of $1 \sigma \sim 3.5 \mu \mathrm{Jy}$ at $\lesssim 5^{\prime}$ from the pointing centre, twice the

\footnotetext{
2 http://www . radionet-eu.org/

3 We assume an empty Friedmann universe $\left(\Omega_{0}=0\right)$, for consistency with Padovani et al. (2004) and take $H_{0}=70 \mathrm{~km} \mathrm{~s}^{-1} \mathrm{Mpc}^{-1}$.
} 


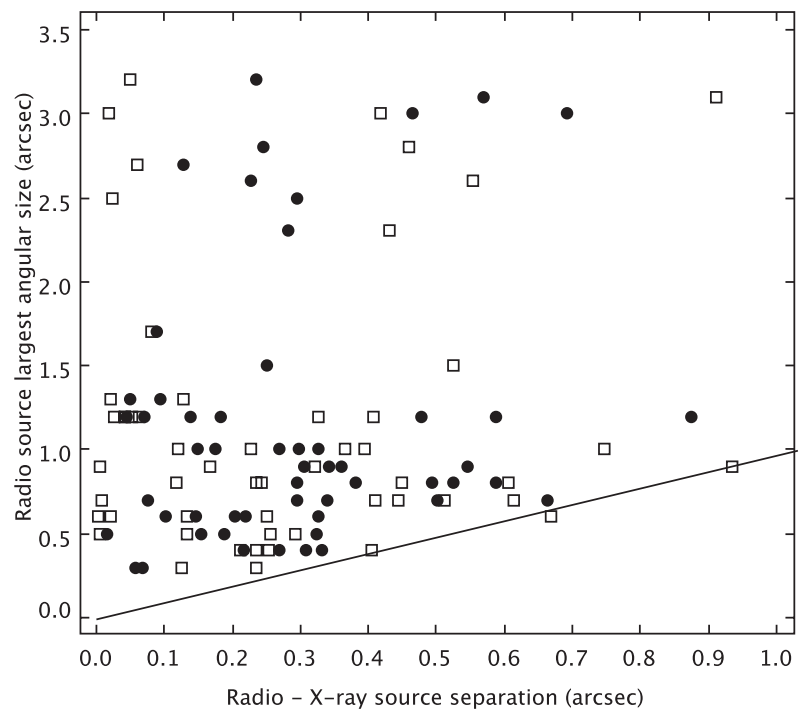

Fig. 1. The filled symbols show the measured angular sizes of radio sources with X-ray counterparts compared with the radio-X-ray peak separation. The sloping line has a gradient of unity, showing that all the radio sources have an angular size greater than the distance to their $\mathrm{X}$-ray counterpart. The hollow symbols show the measured angular size as a function of randomised X-ray position errors (see text).

sensitivity of the VLA-only data. Both arrays observed in widefield mode, using short integration times and multiple narrow frequency channels across the bandpass in order to ensure that time-averaging and chromatic aberrations were less significant than the fundamental limitations of the primary beams. This is described in detail by Richards (2000, his Sect. 3.2 and Fig. 3) and Muxlow et al. (2005, their Sect. 2). Computational limitations meant that the calibrated MERLIN and VLA data were separately Fourier transformed into multiple small dirty maps covering the region to be imaged; each pair was then combined and CLEANed. Tests showed that, for an image with the same weighting and CLEANing, there was no appreciable difference between this method and data combination in the visibility plane (Muxlow et al. 2005, their Fig. 1). The final combined images show $\$ 6 \%$ loss of flux at $5^{\prime}$ from the pointing centre and there is no systematic radial distortion of the source contours (Muxlow et al. 2005, their Fig. C1).

Muxlow et al. (2005) resolved all 92 radio-bright sources at $0.2-2^{\prime \prime}$ resolution, see Table 1 , Fig. 1 and Muxlow et al. (2005). J123644+621133 is an FR 1 (Fanaroff \& Riley 1974) radio galaxy with jets extending over $12^{\prime \prime}$. Excluding this source, the mean angular size of sources at $z \lesssim 2$ is 1 .' 3 , corresponding to $\sim 10 \mathrm{kpc}$ at $z>0.8$. Sources at $2 \lesssim z \lesssim 3$ have a mean size of $8 \mathrm{kpc}$ and the source at $z=4.424$ has a size of $2 \mathrm{kpc}$. The smaller apparent size of higher redshift sources is probably at least partly due to the non-detection of fainter extended emission (as well as being affected by the adopted cosmology) and is not obviously linked to the inverse relationship between angular size and redshift established for bright radio galaxies by Barthel \& Miley (1988).

VLA observations at $8.4 \mathrm{GHz}$ covered the inner $\operatorname{HDF}(\mathrm{N})$ to a radius of $\sim 4^{\prime}$ (Richards et al. 1998; Fomalont et al. 2002) at a resolution of $33^{\prime \prime} 5$, finding a total of 50 sources within the 10 -arcmin field. 27 of these sources were detected at $>40 \mu \mathrm{Jy}$ by MERLIN+VLA at $1.4 \mathrm{GHz}$. The remainder cannot be classified using their radio morphologies and are omitted from our analysis, apart from 7 which do have X-ray counterparts (Sect. 2.2). We refer to these as $8.4-\mathrm{GHz}$ selected sources. Their properties are given in Table 1, including 1.4-GHz flux densities taken from Richards et al. (1998) where available or calculated using the spectral indices described in Sect. 2.1.2, so that the rest-frame luminosities of the whole sample can be derived consistently in Sect. 4.1.

We use $S_{\mathrm{R}}$ to denote the total radio flux density measured by the VLA at either frequency, further subscripted by the specific frequency only where relevant.

Four of the 92 sources were detected at (4-20)-mas resolution by the EVN (European VLBI Network) and global VLBI (Garrett et al. 2001; Chi et al. 2006). At the other extreme, the Westerbork Synthesis Radio Telescope at $15^{\prime \prime}$ resolution detected $\sim 10 \%$ more sources than the VLA (Garrett et al. 2000). Further VLA images on larger scales are in preparation (Morrison et al. 2006) and recent low-frequency observations have been made using the GMRT (Lal, D. V., in prep.).

\subsubsection{Radio spectral indices}

The radio spectral index $\alpha$ is given by

$S_{\mathrm{R}} \propto v^{-\alpha}$.

Asymmetric uncertainties are represented by e.g. $\alpha_{-\sigma_{\alpha_{-}}}^{+\sigma_{\alpha_{+}}}$. Richards (2000) and Richards et al. (1998) provide spectral indices and uncertainties for sources detected by the VLA at both frequencies. If sources within the overlapping spatial region were detected at $1.4(8.4) \mathrm{GHz}$ only, $\alpha$ is a lower (upper) limit depending on the local noise in the 8.4 (1.4) $\mathrm{GHz}$ field. In the case of a 1.4-GHz-only detection $\sigma_{\alpha_{-}}$is approximated as twice the error due to the $1.4 \mathrm{GHz}$ detection,

$\sigma_{\alpha_{-}}=\frac{2}{\ln [8.4 / 1.4]} \frac{\sigma_{S_{\mathrm{R} 1.4}}}{S_{\mathrm{R} 1.4}}$

and $\sigma_{\alpha_{+}}$is given by an analogous expression for 8.4-GHz-only detections.

Radio sources classified as AGN or as starbursts (see Sect. 5) detected at both frequencies had spectral indices in the ranges $(-0.4<\alpha<1.4)$ and $(0.3<\alpha<1.7)$ respectively; all unclassified sources had $\alpha$ within the extrema of these ranges. Where $\alpha$ is a lower limit we set $\sigma_{\alpha_{+}}$to the relevant upper limit. e.g. $(1.7-\alpha)$ for starburst or unclassified sources. The errors in $\alpha$ for 8.4-GHz selected sources were deduced in a similar fashion for the opposite limits. For sources outside the $8.4 \mathrm{GHz}$ field we adopted typical values of $\alpha$ of 0 and 0.8 for AGN and starbursts respectively and an average of 0.4 for unclassified sources, using the extrema to deduce the uncertainties, so that for example a starburst would have $\alpha=0.8 \pm_{0.5}^{0.9}$.

\subsection{X-ray data}

The Chandra X-ray observatory made a total of 2 Ms multiband exposures of the HDF(N) (Alexander et al. 2003b). All $\mathrm{X}$-ray flux densities, counts and luminosities given in this paper refer to the Chandra full band from $0.5-8.0 \mathrm{keV}$ unless otherwise stated. Soft-band values are used for J123709+620841 and $\mathrm{J} 123646+621445$ as they were not detected in the full band. There are 100 sources in common within the area of overlap between the whole VLA and Chandra fields of view, with a median offset of $\sim 0$ !' 2 after small corrections to align the X-ray frame (Alexander et al. 2003b). 


\subsubsection{X-ray - radio counterparts}

The Chandra observations completely enclose the radio 10 -arcmin field and the decline in sensitivity in both images towards the edges of this region is less than $6 \%$. This field contains $253 \mathrm{X}$-ray sources with position uncertainties 0.'3-0!'9. Fiftyfive $(60 \%)$ of the radio-bright sources have X-ray counterparts within $0{ }^{\prime} 9$ of the radio peak; the separation is $<0$. ' 4 for 42 of these. Increasing the cross-match search radius up to $2^{\prime \prime}$ failed to produce any more matches. One or two additional matches appear for each additional arcsec radius from $2-5^{\prime \prime}$. Each of these radio sources also has a counterpart at $<00^{\prime} 9$; in about half these cases the multiple associations appear to be genuine (e.g. similar redshifts). We consider that we can only be confident that the emission is coming from the same galaxy for the 55 unambiguous matches at $<00^{\prime} 9$ separation. These make up $22 \%$ of the X-ray detections in the 10 -arcmin field. Seven additional 8.4-GHz selected sources have X-ray counterparts within their combined position uncertainties.

We compared the largest angular size of each radio-bright source with the X-ray - radio source separation, represented by the solid circles in Fig. 1 (J123644+621133, with an angular size of $12^{\prime \prime}$, has been omitted). In every case the X-ray peak is no further from the radio peak than the most extended radio emission. We produced randomised $\mathrm{X}$-ray position errors, in a Gaussian distribution, such that $80-90 \%$ of the X-ray positions were within the published errors of 0.'3-0.'9 (Alexander et al. 2003 b), which are plotted as hollow squares. The radio peak position errors are negligible in comparison $\left.(\lessgtr 0)^{\prime} 1\right)$. There is no evidence for any systematic excess in the measured source separations with respect to the X-ray position errors but peak offsets of $\lesssim 1^{\prime \prime}$ cannot be ruled out.

\subsubsection{X-ray photon indices}

The X-ray photon index $\Gamma$, for flux density $F_{\mathrm{X}}$ in $10^{-18} \mathrm{~W} \mathrm{~m}^{-2}$ at energy $E \mathrm{keV}$ is defined by

$F_{\mathrm{X}}(E) \propto E^{-\Gamma}$

and $\Gamma$ is equivalent to $\alpha+1$ (where $\alpha$ is the spectral index). All values measured by Alexander et al. (2003b) lie in the range $-1 \lesssim \Gamma \lesssim 2$, who give upper and lower bounds to the uncertainty, $\sigma_{\Gamma_{+}}$and $\sigma_{\Gamma_{-}}$only for sources detected in more than one subband. If a source is only detected in the full and soft bands then $\Gamma$ is a lower limit so we take $\sigma_{\Gamma_{+}}=2-\Gamma$ and approximate $\sigma_{\Gamma_{-}}$ as twice the error due to the uncertainty in the soft band counts. If a source is only detected in the full and hard bands then $\Gamma$ is an upper limit so we take $\sigma_{\Gamma_{-}}=\Gamma-(-1)$ and approximate $\sigma_{\Gamma_{-}}$as twice the error due to the uncertainty in the hard band counts. If sources were only detected in the full band, Alexander et al. (2003b) give an estimated value of $\Gamma=1.4$ and we adopt $\sigma_{\Gamma_{-}}=\sigma_{\Gamma_{+}}=0.6$. In all cases we constrain $\sigma_{\Gamma_{-}} \leq 0.6$ in order to avoid non-physical negative limits on $L_{\mathrm{X}}$ (see Sect. 4.2).

\subsection{Hubble Space Telescope observations}

The original HDF and surrounding fields (out to a distance of $\sim 5^{\prime}$ ) was observed by the HST WFPC2 in 1996 (Williams et al. 1996). In 2003 the GOODS project used the HST ACS to reobserve the HDF region in the $F 435 W, F 606 W, F 775 W$ and $F 850 L P$ filters ( $B, V, i$ and $z$ bands). We find that the GOODS images and source catalogue r1.1z (Giavalisco et al. 2004) require a linear shift of -0.342 in Declination to align them with the ICRF.

\subsection{IR and sub-mm sources}

The ISO fields and the Spitzer catalogue published by Teplitz et al. (2005) only cover part of the 10-arcmin field so it is only possible to give meaningful statistics for the fractions of the IR catalogues detected at other wavelengths (not vice versa). More quantitative analysis will be available using further Spitzer results at $24 \mu \mathrm{m}$ (see e.g. Beswick et al. 2006). Extensive SCUBA searches have been made over most of the $\operatorname{HDF}(\mathrm{N})$.

\subsubsection{Mid-infrared detections}

One hundred sources were detected in the inner $\operatorname{HDF}(\mathrm{N})$ by ISO at 7 or $15 \mu \mathrm{m}$ (Aussel et al. 1999). Although the beam size was $3-6$ " the tight correlation between radio and $15 \mu \mathrm{m}$ flux densities out to at least $z=3$ (Garrett 2002; Elbaz et al. 2002; see Sect. 1) supports the association of radio and IR sources within the position errors even if they overlap more than one optical source. 28 radio-bright sources lie within the $I S O$ field, of which 17 have ISO counterparts (Muxlow et al. 2005). All matched sources were detected at $15-\mu \mathrm{m}$ except for $\mathrm{J} 123656+621301$. This is nonetheless an extended diffuse radio source with a very steep spectrum characteristic of a starburst. An additional 7- $\mu \mathrm{m}$ source in the catalogue of Goldschmidt et al. (1997) is matched with the FR 1 J12364+621133.

The radio-MIR association has been reinforced by recentlypublished Spitzer observations at $16 \mu \mathrm{m}$ (Teplitz et al. 2005). 18 Spitzer sources have MERLIN+VLA counterparts within 1 '.2. Half of these lie outside the ISO fields. Of the other nine, 7 already had ISO counterparts (including the very red source J123651+621221 at $z=2.71$; Teplitz et al. (2005) associate the IR emission with an elliptical galaxy at a similar separation but lower redshift). The other two, J123633+621005 and $\mathrm{J} 123708+621056$, lie close to the edges of the $I S O$ field where its noise was higher. We cannot confidently associate J123646+621445 with the Spitzer source 1."7 to the SW as they have two separate optical counterparts. An ISO source lies within $3^{\prime \prime}$ of the $16 \mu \mathrm{m}$ source but further from J123646+621445. There are no further candidate radio-IR matches within $2^{\prime \prime}$.

The combined Spitzer and ISO data contain 205 separate 15or $16-\mu \mathrm{m}$ sources within the 10 -arcmin field of which a quarter (53) have X-ray counterparts. Even fewer $(26,13 \%)$ have radiobright counterparts, but almost all of these (21/26) are also X-ray detections. This complements the tendency, noted by Alexander et al. (2002), that optically identified (emission line) $15 \mu \mathrm{m}$ starbursts with X-ray emission are more likely to have radio counterparts than those without. Four of the $78.4-\mathrm{GHz}$ selected sources with X-ray counterparts have ISO counterparts, 3 of which were also detected by Spitzer.

\subsubsection{SCUBA detections}

Several sets of observing and data reduction techniques have produced various SCUBA catalogues optimised for different regions and properties (e.g. Serjeant et al. 2003; Borys et al. 2004; Wang et al. 2004; Chapman et al. 2005). The techniques used to minimise ambiguity in cross-identifications are summarised in Muxlow et al. (2005). The most comprehensive list is currently provided by Borys et al. (2004) (the revisions by 
Pope et al. 2005, do not affect any radio-bright sources). We use all their secure identifications between SMGs and radiobright sources. We also include the additional identifications of $\mathrm{J} 123622+621629$ and $\mathrm{J} 123711+621325$ made by Chapman et al. (2005). We do not include the SMGs known as HDF 850-1 and 850-6 as most authors conclude that they do not have radiobright counterparts. J123608+621431 is $\sim 3^{\prime \prime}$ from the nearest $\mathrm{X}$-ray source so it is not included in the detailed analysis in this paper, but both objects are within the larger error circle of a SCUBA source. We reject the identification of J123646+621445 for reasons similar to those given in Sect. 2.4.1 with respect to IR sources.

This leaves 16 radio-bright sources in the 10 -arcmin field with SCUBA counterparts, of which 11 were also detected by Chandra; one further $8.4-\mathrm{GHz}$ source has both SCUBA and $\mathrm{X}$-ray detections. All these sources have either spectroscopic or photometric redshifts, which we adopt in preference to redshifts derived from the $1.4-\mathrm{GHz} / 850-\mu \mathrm{m}$ flux density ratio in order to avoid circular arguments.

\subsection{Redshifts}

61 radio-bright sources and 140 Chandra sources in the 10 -arcmin field have measured redshifts, including 50 of the 55 radio-bright $\mathrm{X}$-ray sources. 19 of the $8.4-\mathrm{GHz}$ selected sources also have measured redshifts, including 7 with X-ray counterparts.

Table 1 gives our adopted redshift measurements, uncertainties $\sigma_{\mathrm{z}}$ and references for the sources detected in both regimes. We include the published errors, $\sigma_{z}$, where given. If not we adopt $\sigma_{z}=0.003$ for spectroscopic redshifts, which were all obtained using the Keck LRIS or instruments with resolution as good or better. The uncertainties in photometric redshifts are $\leq z / 4$ apart from $\mathrm{J} 123725+621128$ where $1<z<2$ was estimated from the $K: z$ band flux density ratio (Hornschemeier et al. 2001). The uncertainties do not include possible misidentifications of objects or of spectral lines, nor instabilities in photometric fitting. In most cases the differences between different redshift estimates for the same source are small, or have been discussed and resolved in the literature. The redshift for J123616+621513 has now been revised to 2.58 (Chapman et al. 2004a). We adopt recently-published redshifts for faint NICMOS or ACS galaxies associated with the radio sources J123606+621021, $\mathrm{J} 123642+62133, \mathrm{~J} 123651+621221$ and $\mathrm{J} 123716+621512$, in preference to the photometric redshifts derived by Barger et al. (2003) for their X-ray counterparts using more widely separated, older optical detections.

The redshift distributions of radio and X-ray sources and of objects detected in both regimes are compared in Fig. 2. All three distributions peak at $0.5<z<1.0$ but the fraction of X-ray sources with measured redshifts which are radio-loud changes from less than a third at $z<1$ to a half or greater at higher $z$. A similar increase in codetections with redshift is seen in the fraction of radio sources which are X-ray-selected type-II AGN. We used the Kolmogorov-Smirnov test to investigate the relationship between the redshift distributions of the radio and X-ray sources. We found that there is a $93 \%$ probability that radio and $\mathrm{X}$-ray sources at $z<1.1$ are drawn from the same population and a $98 \%$ probability for sources at $z>1.1$, but this drops to an insignificant probability of $27 \%$ for all redshifts considered together. This only makes sense if the radio counterparts to X-ray sources at lower redshifts are a separate population from those at higher redshifts. These implications are discussed in Sects. 5.2 and 7.

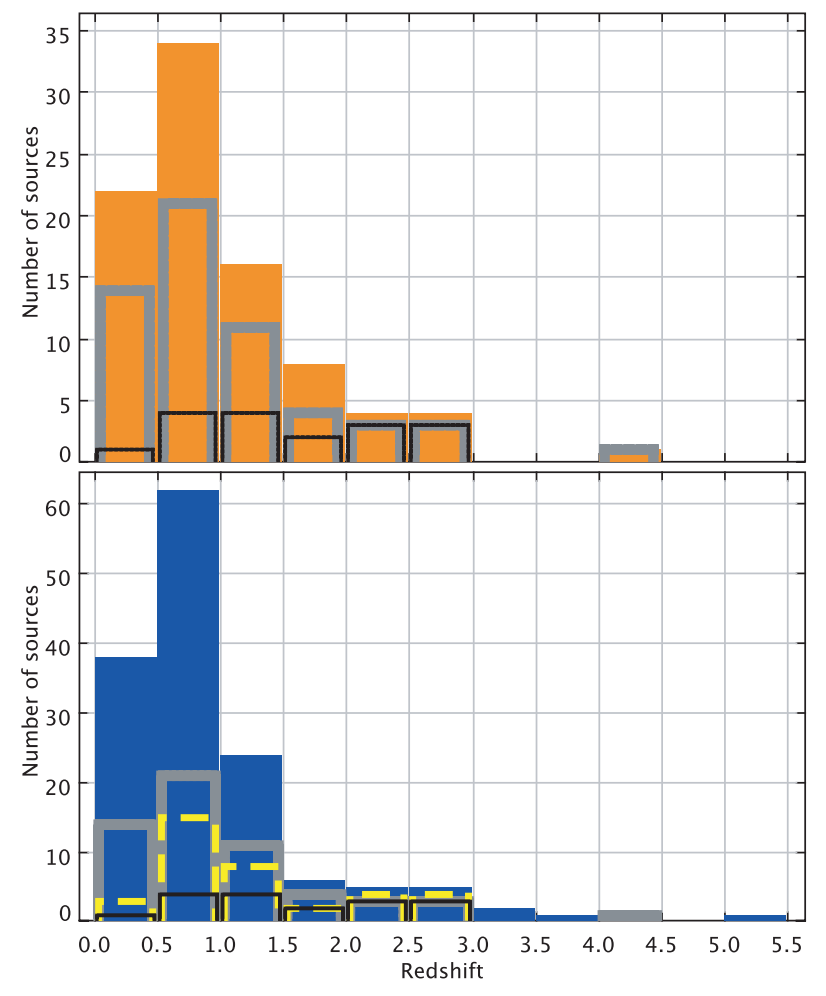

Fig. 2. The distribution of sources with published redshifts in the 10 -arcmin field. The filled blue area in the lower panel shows X-ray sources and the dashed yellow line shows X-ray selected type-II AGN. The filled orange area in the upper panel shows radio sources. In both panels the cross-matched X-ray and radio sources, and those which are also X-ray selected type-II AGN, are shown by the thick grey and thin black lines, respectively. (This figure is available in color in electronic form.)

\section{New technology}

\subsection{Virtual Observatory facilities}

We made use of a wide range of published surveys and catalogues. These were obtained using the Vizier ${ }^{4}$ service where possible, in order to select sources in the exact area covered by the radio data, and obtain tables in VOTable ${ }^{5}$ format for ease of further manipulation. Other data (e.g. in IPAC format) were converted to VOTable using TopCat ${ }^{6}$, which preserves accuracy equivalent to full double precision. Sources were crossmatched using either the AstroGrid ${ }^{7}$ Xmatch tool or TopCat, which allowed us to identify and correct for any systematic linear offsets due to astrometric errors. We also used TopCat to calculate luminosities and other derived quantities (Sect. 4) presented in the tables, and to prepare many of the plots.

The original images have resolutions from $\sim 0.015$ (HST) to several arcsec (ISO, SCUBA). The MERLIN+VLA and HST maps are made up of many small panels each containing about a million pixels. We used the Aladin visualisation tool as modified for the Euro- $\mathrm{VO}^{8}$ to find, cross-identify and visualise regions of interest on such different scales; an example is shown in Fig. 3. The International Virtual Observatory ${ }^{9}$ Simple Image

\footnotetext{
4 http://vizier.u-strasbg.fr/

5 http://cdsweb.u-strasbg.fr/doc/VOTable/

6 http://www.starlink.ac.uk/topcat

7 http://www.astrogrid.org

8 http://www. euro-vo.org

9 http://www.ivoa.net
} 


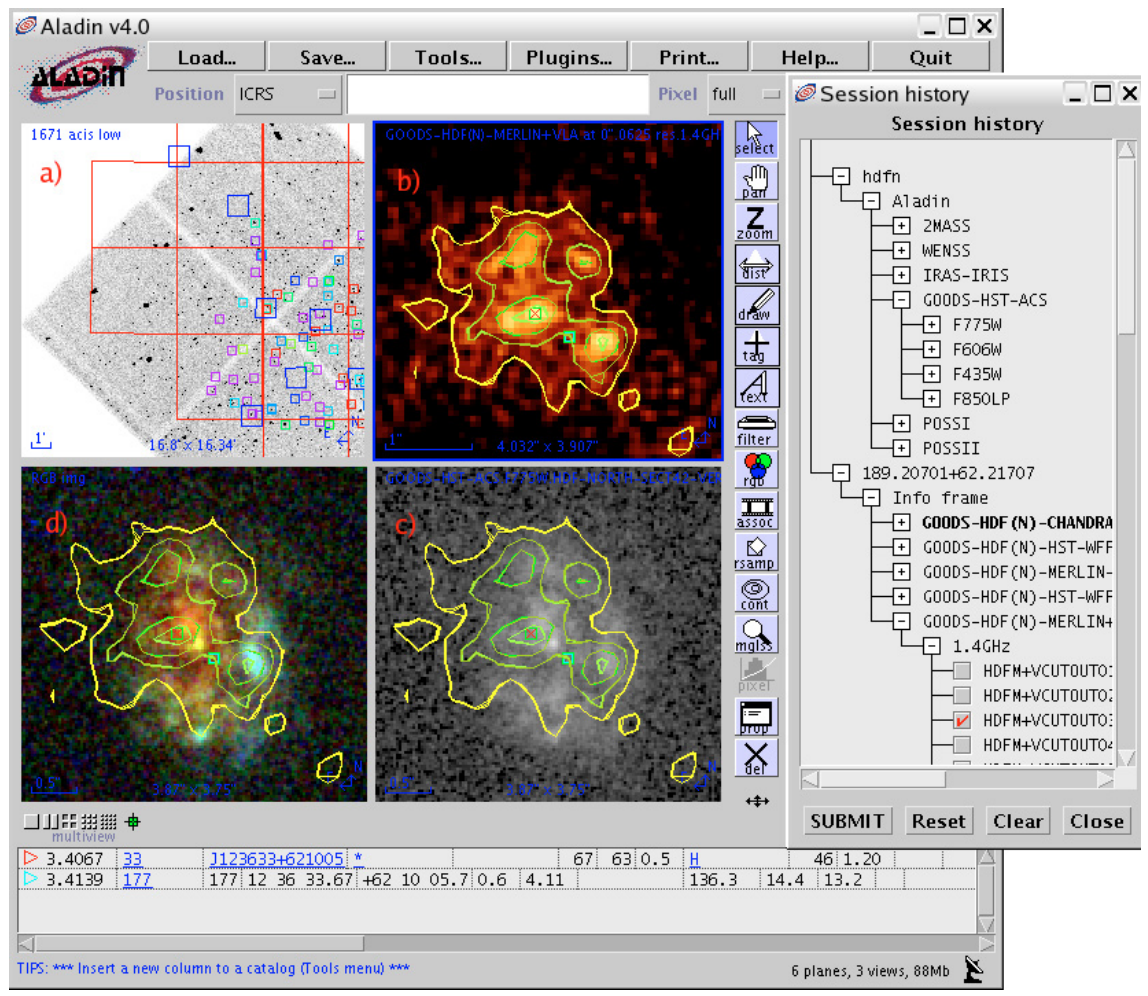

Fig. 3. The use of the Euro-VO Aladin and PLASTIC to investigate the starburst candidate J123633+621005. Clockwise from top left, the panels show: a) Chandra image overlaid with the outlines of HST ACS field boundaries; clicking in the appropriate square selects the relevant image(s) for loading. The coloured symbols show radio sources, with symbol size proportional to source size and shade proportional to redshift. b) Radio contours for $\mathrm{J} 123633+621005$; the red cross and blue square mark radio and X-ray peaks, respectively. c) $A C S F 775 W$ image d) $A C S$ false colour composite of $F 435 W, F 606 W$ and F850LP bands, all overlaid with radio contours. The scale bars in the bottom left of each panel represent a) $1 \mathrm{arcmin}$, b) 1 arcsec, c) and d) 0.'5. (This figure is available in color in electronic form.)
Access Protocol (SIAP) for descriptions of images and their locations is used to locate the corresponding fields in Chandra, MERLIN+VLA and HST images despite the different resolutions, image sizes and even orientations. The PLASTIC ${ }^{10}$ protocol developed for VOTech ${ }^{11}$ allows any of these VO tools to manipulate the same data.

\subsection{RadioNet tools and dynamic radio imaging}

It is now feasible to image much larger radio fields in entirety, compared with the epoch when the MERLIN+VLA observations were made, thanks to increased computing power, improved algorithms in AIPS and the use of Virtual Observatory standards and tools for data management. We made 81 slightly overlapping square images, each of $1024 \times 0$. .'0625 pixels on a side. We combined these images into a single $(8 \times 8) \mathrm{arcmin}^{2}$ 1.4-GHz image, hereafter the 8 -arcmin field. This contains over 67 Mpixels, covering most of the maximum sensitivity regions of both the radio and X-ray images, with good overlap with the $A C S$ data. This will be extended to cover the 5-arcmin radius region of near-optimum radio sensitivity. We describe our use of the new 8-arcmin $\mathrm{HDF}(\mathrm{N})$ image in Sect. 8.

The range of baseline lengths in the combined MERLIN+VLA data means that maps can be extracted at resolutions of $0.2-22^{\prime \prime}$ depending on whether the observer wants to investigate potential compact hot spots or faint extended emission. Muxlow et al. (2005) gives a full description of the method which was used to produce the earlier hand-processed images. We now provide an automatic imaging service which extracts the required region and convolves it with the chosen restoring beam within this resolution range. This uses the python-based package ParselTongue ${ }^{12}$ (Kettenis et al. 2006),

\footnotetext{
10 http://plastic.sourceforge.net/

11 http://eurovotech.org/

12 http://www.radionet-eu.org/rnwiki/ParselTongue
}

developed in the RadioNet Consortium, to provide a scripting interface between AstroGrid and "classic" AIPS. The AstroGrid workbench offers a simple dialogue box for the user to select image size, resolution and region within the $\operatorname{HDF}(\mathrm{N})$. These parameters are passed to the MERLIN archive server which uses ParselTongue to extract the required image. A pointer to the image and a basic (SIAP-compliant) description is either returned straight to the user or can be used to pass it to another VO-enabled tool such as Aladin or a source extractor. This VO tool and the complementary MERLINImager (which operates on the visibility data for other MERLIN archive data) are the first to allow an astronomer to obtain customised radio images without having to install their own specialised radio data reduction package. Moreover, only the required image (at most $0.25 \mathrm{~GB}$ ) is moved over the internet to the point of use; the parent data set, which can be many GB, is processed in situ.

\section{Estimation of radio and X-ray luminosities}

There is no correlation between radio and X-ray flux densities for the whole cross-matched sample nor for any subsets; however this is not surprising given the wide span of redshifts and the different behaviour of the spectral/photon indices for different sources. We therefore compared the $K$-corrected luminosities for all 50 sources with measured redshifts.

\subsection{Rest frame 1.4-GHz radio luminosity}

Table 1 lists the observed-frame 1.4-GHz total radio flux density per source, $S_{\mathrm{R}}$, in $\mu \mathrm{Jy}$. The flux densities and their uncertainties $\left(\sigma_{S_{\mathrm{R}}}\right)$ are given in Muxlow et al. (2005) and Richards et al. (1998).

We assume an empty Friedmann universe $\left(\Omega_{0}=0\right)$, for consistency with Padovani et al. (2004) and take $H_{0}=$ $70 \mathrm{~km} \mathrm{~s}^{-1} \mathrm{Mpc}^{-1}$. The radio rest-frame luminosity $L_{\mathrm{R}}$, taking 
into account the $K$-correction and the expansion of the bandwidth in the observed frame, is given in $\mathrm{W} \mathrm{Hz}^{-1}$ by

$L_{\mathrm{R}}=\frac{S_{\mathrm{R}}}{10^{32}} 4 \pi\left(d \times 3.086 \times 10^{22}\right)^{2}(1+z)^{\alpha-1}$

where $d$ is the luminosity distance in kpc, given by

$d=\frac{c z}{H_{0}}(1+z / 2)$

where the speed of light, $c$, is in $\mathrm{km} \mathrm{s}^{-1}$. The lower bound on the uncertainty $\sigma_{L_{R}-}$ is given by

$\sigma_{L_{\mathrm{R}}-}=\sqrt{\sigma_{L_{\mathrm{R}}(\alpha-)}+\sigma_{L_{\mathrm{R}}\left(S_{\mathrm{R}}\right)}+\sigma_{L_{\mathrm{R}}(z)}}$

where the partial errors in $L_{\mathrm{R}}$ due to the lower bound on the spectral index error, due to the flux density error and due to the redshift error are given by:

$\sigma_{L_{\mathrm{R}}(\alpha-)}=L_{\mathrm{R}} \sigma_{\alpha_{-}} \ln (1+z)$

$\sigma_{L_{\mathrm{R}}\left(S_{\mathrm{R}}\right)}=L_{\mathrm{R}} \frac{\sigma_{S \mathrm{R}}}{S_{\mathrm{R}}}$

$\sigma_{L_{\mathrm{R}}(z)}=L_{\mathrm{R}} \sigma_{z}\left(\frac{2(1+z)}{z(1+z / 2)}+\frac{\alpha-1}{1+z}\right)$.

Substituting $\sigma_{\alpha_{+}}$for $\sigma_{\alpha_{-}}$in Eq. (8) gives $\sigma_{L_{\mathrm{R}}(\alpha+)}$ which then replaces $\sigma_{L_{R}(\alpha-)}$ in Eq. (7) to give the upper bound to the uncertainty in $L_{\mathrm{R}}, \sigma_{L_{\mathrm{R}}+}$. Table 2 gives $L_{\mathrm{R}}$ and its upper and lower bounds $\left(L_{\mathrm{R}}-\sigma_{L_{\mathrm{R}}-}\right.$ and $\left.L_{\mathrm{R}}+\sigma_{L_{\mathrm{R}}+}\right)$.

\subsection{Rest-frame $0.5-8.0 \mathrm{keV} X$-ray luminosity}

The rest-frame X-ray luminosity is given by the analogy of Eq. (5).

$L_{\mathrm{X}}=\frac{F_{\mathrm{X}}}{10^{18}} 4 \pi\left(d \times 3.086 \times 10^{22}\right)^{2}(1+z)^{\Gamma-2}$.

The observed flux densities are given in Table 1, taken from Alexander et al. (2003b). This does not give flux density uncertainties so we assume that the relative uncertainty in the flux density is equivalent to the relative uncertainty in the counts $(C)$. $\sigma_{\mathrm{C}-}$ and $\sigma_{\mathrm{C}+}$ are the lower and upper bounds on the uncertainties in the counts. The lower limit to the luminosity uncertainty $\sigma_{L_{X}-}$ is given by the sum in quadrature of uncertainties due to the lower limits to the errors in $\Gamma$ and $F_{\mathrm{X}}$ and to the error in $z$

$\sigma_{L_{X}(\Gamma-)}=L_{X} \sigma_{\Gamma-} \ln (1+z)$

$\sigma_{L_{X}\left(F_{X^{-}}\right)}=L_{X} \frac{\sigma_{C-}}{C}$

$\sigma_{L_{X}(z)}=L_{X} \sigma_{z}\left(\frac{2(1+z)}{z(1+z / 2)}+\frac{\Gamma-2}{1+z}\right)$

and the upper limit to the uncertainty, $\sigma_{L_{X}+}$, is given by an analogous expression. Table 2 gives $L_{\mathrm{X}}$ and its upper and lower bounds $\left(L_{X}-\sigma_{L_{X}-}\right.$ and $\left.L_{X}+\sigma_{L_{X}+}\right)$.

\section{Origins of radio and X-ray emission}

In this section, we discuss diagnostics for the specific origins of the radio and X-ray emission, based on the references and discussion of Sect. 1, applied to the derived source properties. We keep the initial radio source classification independent of X-ray properties (and vice versa) as we wish to investigate whether the observed radio and X-ray emission comes from different sources within the same galaxies. In particular, we do not use published radio-X-ray relationships such as Eq. (1) for classification, but compare our results with this in the next Sect. 6.

\subsection{The nature of the radio emission}

The main diagnostics for the origins of the radio-bright emission are:

1. Morphology: The presence of a bright, compact core or clear radio jets/lobes suggests the presence of a radio AGN. Extended emission (kpc scales) with no obvious peaks or jets/lobes is more likely to originate from star formation.

2. Spectral Index: Radio emission with $\alpha \lesssim 0.4$ is likely to be powered by an AGN. Steeper-spectrum emission is usually of starburst origin but could indicate a lobe-dominated AGN system, hence the need to inspect the structure.

3. The Radio-FIR link: Rest-frame FIR or MIR emission bright enough to be detectable in the HDF(N) (including by SCUBA, for high- $z$ sources) indicates a ULIRG-like intensity or greater, very likely to be of starburst origin, found to be closely correlated with radio emission. Even when there is separate evidence for the presence of an AGN (see Sect. 1), rest-frame MIR emission at or greater than ULIRG-like luminosity is far in excess of the predictions of any known AGN model (Alexander et al. 2005a, see also Sect. 7.2) and AGN are unlikely to be responsible for more than $20 \%$ of the bolometric luminosity (Alexander et al. 2003a, 2005b).

4. Optical appearance: If bright radio and optical galaxy cores coincide, this is further evidence for the presence of an AGN. Extended radio emission outside the apparent optical extent of a galaxy is likely to be of jet origin. On the other hand, extended radio emission within the optical galaxy, especially if associated with bright optical knots, indicates active star formation. Very disturbed or interacting galaxies with associated diffuse radio emission provide supporting evidence of conditions for starburst activity. As explained in Sect. 1, obscuration can hide optical signatures, especially in starbursts, so absence of (optical) evidence is not conclusive evidence of absence of a particular radio emission mechanism.

Muxlow et al. (2005) described in detail the application of these criteria in classifying radio sources as Starburst (SB), Active Galactic Nucleus (AGN), or unclassified (U). Note that the AGN status covers any emission powered by an AGN, whether it arises from accretion or from jets/lobes. Using the MERLIN+VLA data, we are able to distinguish between extended lobes and cores, but not between pc-scale jets and the core itself, although the resolved emission of AGN origin must be from jets or lobes. The presence of two unambiguous FR 1 in the $\operatorname{HDF}(\mathrm{N})$ already represents a greater space density than would be expected from local number counts (Snellen \& Best 2001), making it unlikely that a high proportion of the unidentified extended or steepspectrum sources have large radio lobes.

VLBI results support the AGN interpretation of compact, flat-spectrum radio cores. Extremely compact radio cores (brightness temperature $\left(>10^{5}-10^{6}\right) \mathrm{K}$ ) were detected in $\mathrm{J} 123642+621331, \mathrm{~J} 123644+621133, \mathrm{~J} 123646+621404$ and $\mathrm{J} 123652+121444$ using the European VLBI Network (EVN) and global VLBI (Garrett et al. 2001; Chi et al. 2006), confirming the presence of an AGN. J123644+621133 is unmistakably an FR 1. 
The EVN recovers all the VLA flux from J123646+621404 About $1 / 3$ of the VLA flux from J123652+121444 is present in the 4-mas resolution global VLBI image but the source is known to be variable (Richards et al. 1998). All three sources have flat or inverted spectra. J123642+621133 is discussed in more detail in Sect. 5.3; in summary we infer that it consists of compact AGN-powered emission embedded in a more diffuse starburst. The MERLIN+VLA data suggest that this is also the situation for J123635+621424 and J123642+121545.

Table 2 gives our classifications for the objects with X-ray counterparts. The recent ACS and Spitzer data and improved SCUBA source lists have allowed us to strengthen the classification of a number of sources. We have changed the classification of 4 sources, as follows. J123622+621544 was tentatively assigned AGN status by Muxlow et al. (2005) but the $A C S$ image shows that the radio emission is extended over bright optical knots in a distorted spiral, not seen in the original CFHT plate (Canada-France-Hawaii Telescope, Barger et al. 1999). It is also a new MIR detection by Spitzer and has a radio spectral index $>0.6$ so we reclassify J123622+621544 as a starburst. We infer from the Spitzer and ACS images that two previously unclassified sources with steep radio spectra are starbursts. J123629+621046 is extended, with a red optical counterpart which is either a distorted galaxy with a dust lane or two interacting galaxies. J123641+620948 has a compact core but the ACS image confirms the suggestion by Cohen et al. (2000) that it is associated with two interacting spiral galaxies. The ACS morphology allows us to reclassify J123642+621545 as a starburst candidate with a possible AGN core, as its extended radio emission overlays blue knots in the arms of a face-on spiral. It also possesses a relatively bright compact radio and optical core. It was detected by $I S O$ and has an intermediate radio spectral index of 0.5 .

Note that the classifications of radio-bright sources are made primarily on the basis of radio properties such as morphology and/or spectral index (conditions 1. and 2. above) whilst information from other wavebands (conditions 3. and 4.) is used as supporting evidence. The origins of the radio emission from the seven 8.4-GHz-selected sources with X-ray counterparts are less certain as they are unresolved in the radio and have approximate spectral indices or upper limits only. The ACS images show that $\mathrm{J} 123637+621135, \mathrm{~J} 123639+621249$ and $\mathrm{J} 123648+621427$ are associated with spiral galaxies with knots of star-formation (the lower-resolution CFHT image of J123648+621427 appeared elliptical). J123644+621249 is associated with a pair of apparently interacting optical galaxies at very similar redshifts. All four have $\alpha \gtrsim 0.6$ and we list them as starbursts although the closest $(\mathrm{J} 123637+621135)$ is in fact of low luminosity, more like a normal star-forming galaxy. The remaining 3 have flatter spectra; $\mathrm{J} 123655+621311$ is associated with an elliptical galaxy likely to contain an AGN; the other two are unclassified.

In total, the 92 radio-bright sources include 23 unclassified objects, 52 starbursts and 17 AGN, using the radio-based classification. The 55 sources with X-ray counterparts include 9 of the unclassified sources, 36 starbursts and 12 radio AGN. The starburst:AGN ratio is almost identical, $\approx 3: 1$, to the that of the full radio-bright sample. The three starbursts which contain radio AGN (counted once only, as starbursts) are all X-ray detections. MIR observations only cover part of the field but contain 22 sources detected at $15-$ or $16-\mu \mathrm{m}$ as well as in the radio and X-ray. Nineteen of these $(86 \%)$ are radio starbursts, including 3 with radio AGN cores, 4 with X-ray selected type-II AGN (see Sect. 5.2) and one with both. Two more are probably AGN, J123646+621404 (also an X-ray type-II AGN) and
J123709+620841 (see Muxlow et al. 2005). J123655+620808 is unclassified as, although the ACS image shows an apparently spiral galaxy with a dust lane, the extended radio emission is misaligned.

\subsection{Origins of $X$-ray emission}

The great majority of X-ray sources in the HDF region are unresolved by Chandra so only luminosity and spectral index information may be available to determine the specific origin of the $\mathrm{X}$-ray emission. Many classifications in the literature are based on optical and other properties which could be due to separate mechanisms within the host galaxy. A comprehensive sourceby-source breakdown is not available but out of the 19 sources from the $1 \mathrm{Ms}$ sample cross-matched by Bauer et al. (2002b), about $1 / 3$ are classed as emission-line galaxies and presumed to have X-ray emission of starburst origin; most of the remainder are X-ray AGN.

Star-forming galaxies and ULIRGs show a close correlation between their star formation rates represented by FIR emission, and both hard- and soft-band X-ray emission (Ranalli et al. 2003), although Rosa-Gonzalez et al. (2007) has recently found that, for a higher-redshift sample in the $\operatorname{CDF}(\mathrm{S})$, the SFR implied hard-band luminosities can be excessive compared from the rates derived from soft-band or Spitzer MIR data, presumably due to obscured-AGN contamination in the hard band. Hard $\mathrm{X}$-ray emission associated with star formation is thought to originate from high-mass X-ray binaries (e.g. Grimm et al. 2003). More slowly-evolving low-mass X-ray binaries are likely to be less significant (Rosa-Gonzalez et al. 2007), especially in young starburst galaxies. This leaves young supernova remnants and hot plasmas associated with star-forming regions and galactic winds as possible sources of the soft-x-ray component (Ranalli et al. 2003), especially if super star clusters are forming (Griffiths et al. 2000), as discussed by Norman et al. (2004).

The X-ray luminosity of most optically classified starbursts is $<10^{35} \mathrm{~W}$ (Alexander et al. 2002) whilst the presence of detectable hard band $(2-8 \mathrm{keV})$ emission and X-ray luminosities $\geq 10^{35} \mathrm{~W}$ is usually taken to indicate the presence of an AGN; $\geq 10^{37} \mathrm{~W}$ implies a QSO (Alexander et al. 2003a). However, it is not unreasonable that the most extreme starbursts could exceed an X-ray power of $10^{35} \mathrm{~W}$, if the X-ray luminosity is proportional to the rest-frame IR emission (e.g. Ranalli et al. 2003), whilst some nearby FR 1 have X-ray luminosities of only $10^{33}-10^{35} \mathrm{~W}$ (Evans et al. 2006). Soft-band dominated X-ray emission (photon index $\Gamma \sim 2$ ) can indicate a starburst origin (Ptak et al. 1999) but is also seen from unobscured AGN (George et al. 2000). In the latter situation the emission could be due to accretion or to jets but both mechanisms are AGN-powered and included in X-ray AGN statistics.

Obscured (type II) AGN have a harder photon index $(\Gamma \lesssim$ 1.0 ); they are the only known sources with $\Gamma \lesssim 0.5$ (Alexander et al. 2005a) but $0.5<\Gamma<1$ is also seen from high-mass X-ray binaries in starbursts. Nonetheless, the combination of $\Gamma \lesssim 1$ with a rest-frame $2-8-\mathrm{keV}$ luminosity $L_{\mathrm{XH}} \geq 10^{35} \mathrm{~W}$ can only be explained by a type-II AGN (see Sect. 6.2). Padovani et al. (2004) identified a total of 91 such sources in the $\operatorname{HDF}(\mathrm{N})$ with a hardness ratio corresponding approximately to $\Gamma \lesssim 1.15$. Of these, 64 lie within the 10-arcmin field and 17 are radio-bright. These are identified in Table 2. A column density $N_{\mathrm{H}}>10^{27} \mathrm{~m}^{-2}$ is required to provide sufficient obscuration. The estimates of $N_{\mathrm{H}}$ given by Alexander et al. (2005a) for SMG confirmed that all 8 
of the type-II AGN common to their sample and ours exceed this threshold.

Bauer et al. (2004) find that around $75-90 \%$ of the 2-Ms $\mathrm{HDF}(\mathrm{N}) \mathrm{X}$-ray sources are AGN, of which about 2/3 appear absorbed, and about half the remainder are starbursts. A variety of studies of the GOODS fields, including the use of multiwavelength properties (Hornschemeier et al. 2001; Bauer et al. 2002b; Szokoly et al. 2004) give similar results, implying a ratio of approximately 8:1 AGN to starbursts among the X-ray detected sources.

Our sample contains $62 \mathrm{X}$-ray sources with radio-bright counterparts of which 17 or 18 appear to be heavily obscured X-ray AGN. In total, 37 (about 2/3) of the sources with measured redshifts, have hard-band X-ray luminosities brighter than $10^{35} \mathrm{~W}$ (see Sect. 6.2) suggesting the presence of an AGN of some type (Alexander et al. 2003a; Cowie et al. 2004a). Statistically, the majority of all the X-ray emission is probably AGN-powered but it is not possible to distinguish between very luminous but softer emission from starbursts or unobscured AGN on the basis of X-ray properties alone; moreover, diagnostics from other regimes do not guarantee that the emission is from the same phenomenon on a sub-galactic scale. We therefore concentrate on comparing the X-ray-selected type-II AGN population with radio sources classified as AGN or as starbursts.

\subsection{The high-redshift source $J 123642+121331$}

The highest redshift radio source, $\mathrm{J} 123642+621331$, at $z=$ 4.424 , has a high total $1.4-\mathrm{GHz}$ flux density $(467 \mu \mathrm{Jy})$. It has a steep radio spectrum, it is a very reddened NICMOS detection (Waddington et al. 1999) and it was detected by $I S O$ at $15 \mu \mathrm{m}$, all properties consistent with starforming activity. Its 1.4-GHz/FIR ratio, however, is 20-50 times higher than other $\operatorname{HDF}(\mathrm{N})$ starforming galaxies (Garrett 2002). The MERLIN+VLA image shows that about $10 \%$ of the flux is diffuse and extended at between 100-200 mas from the core $(\approx 1 \mathrm{kpc})$, which is likely to contain the starburst component. The star formation rate inferred from the IR flux density is $\approx 1000 M_{\odot} \mathrm{yr}^{-1}$, comparable to the other highest star formation rates deduced for starbursts in the $\operatorname{HDF}(\mathrm{N})$, which would account for $\approx 1 \%$ of the radio emission.

The compact core is AGN-dominated; the EVN detected over half the 1.4-GHz flux (Garrett 2002) and global VLBI (Chi et al. 2006) resolves a jet component a few tens of pc from the core. J123642+621331 has a measured $\Gamma=1.35$, above the limit for type-II AGN, but Padovani et al. (2004) noted that high-redshift sources might be misclassified. The expressions in Sect. 4.2 assume that $\Gamma$ is constant from the observed frame to the rest frame. Alexander et al. (2005a), in their Fig. 7, demonstrate how absorption is a strong function of wavelength, such that for $N_{\mathrm{H}} \gtrsim 2 \times 10^{27} \mathrm{~m}^{-2}$, rest-frame energies $\gtrsim 6 \mathrm{keV}$ are much less affected than lower energies. At $N_{\mathrm{H}} \gtrsim 5 \times 10^{27} \mathrm{~m}^{-2}$ the iron emission and absorption lines, at rest frame energies 6-7 keV and 7-8 keV respectively, become more prominent. The measured $\Gamma$ is derived from the ratio of flux densities above and below $2 \mathrm{keV}$ in the observed frame. This dividing energy corresponds to $\geq 6 \mathrm{keV}$ at $z \geq 2$, so the observed $\Gamma$ of a high redshift absorbed source may appear greater than the actual restframe $0.5-8 \mathrm{keV}$ photon index. In turn, the actual $L_{\mathrm{X}}$ would be higher than the value given in Table 2. J123642+621331 would be most strongly affected. If it is a type-II AGN with a rest frame $0.5-8 \mathrm{keV} \Gamma \lesssim 1.15$, this is compatible with the observed $\Gamma=1.35$.

\section{Relationships between $\mathrm{X}$-ray and radio luminosities}

Figure 4 shows the relationship between $L_{\mathrm{R}}$ and $L_{\mathrm{X}}$ taken from Table 2 for the radio-bright $\mathrm{X}$-ray sources with redshifts. The symbol sizes and shapes represent the largest angular size and the classification applied to the radio emission with a blue $\mathbf{A}$ denoting the presence of an X-ray selected type-II AGN. The shade of the symbols indicates the redshift. The accuracy of our estimates of $L_{\mathrm{R}}$ and $L_{\mathrm{X}}$ and potential selection effects are shown in Fig. 5, for all cross-matched sources with redshifts. All sources shown have measured flux densities in both radio and $\mathrm{X}$-ray regimes and measured redshifts (Sect. 2.2 and Table 1). Filled triangles pointing up (down) indicate objects which were detected at both 1.4 and $8.4 \mathrm{GHz}$ (in at least two X-ray bands) giving a measured spectral (photon) index. Thus, the filled stars show objects with complete measurements. Where one band is a defined limit, an arrow shows the resulting direction of uncertainty in the luminosity. Open triangles show sources where the spectral or photon index has been estimated as described in Sects. 2.1.1 and 2.2. The error bars were derived as described in Sect. 4 which also describes the method for estimating spectral (photon) indices where a source was only detected in one band in the radio (X-ray) regime; such sources are shown by open triangles pointing up (down).

Sources with a given rest-frame luminosity can be detected at higher redshifts if they have steeper spectra, implying that the sample might be biased towards radio starbursts with lowobscuration X-ray counterparts. The red lines in Fig. 5 show the limits of detectability by the MERLIN+VLA and Chandra observations described in Sect. 2 for the two arbitrary combinations of $\alpha$ and $\Gamma$. The lines are marked with the redshifts out to which a source would be detectable for the combination of luminosities at that point and the spectral/photon index combination for that line. This shows that X-ray sources at $z \gtrsim 1.5$ need to have $L_{\mathrm{X}} \gtrsim 10^{35} \mathrm{~W}$ (i.e. in the AGN regime) to be detectable if they have harder photon indices.

\subsection{Relationships for starburst-selected sources}

Figure 6 shows that there is a correlation between $L_{X}$ and $L_{R}$ at lower luminosities but there is a very large scatter at $L_{X} \gtrsim$ $10^{35} \mathrm{~W}$, in particular for sources at $z \gtrsim 1.3$. We investigated this by looking for power-law relationships between $\log L_{\mathrm{R}}$ and $\log L_{\mathrm{X}}$ expressed as

$\log L_{\mathrm{X}}=B \log L_{\mathrm{R}}+A$

using values of the intercept and slope, $A_{i}$ and $B_{i}$ such that the derived $i$ th values of $\log L_{\mathrm{X} i}$ and $\log L_{\mathrm{R} i}$ enclosed the observed values of $\log L_{\mathrm{R}}$ and $\log L_{\mathrm{X}}$. We varied $A_{i}$ and $B_{i}$ between 1.0-50.0 and 0.1-5.0, respectively, in increments of 0.1 . In each case, we calculated the chi-squared: $\left[\left(\log L_{\mathrm{R} i}-\log L_{\mathrm{R}}\right)^{2}+\left(\log L_{\mathrm{X} i}-\right.\right.$ $\left.\left.\log L_{\mathrm{X}}\right)^{2}\right] / \epsilon_{i}^{2}$ where $\epsilon_{i}^{2}$ is the combined error taking into account asymmetric errors, e.g. using $L_{\mathrm{R}-}$ where $L_{\mathrm{R}}>L_{\mathrm{R} i}$ and so on. We attempted to find values of $A$ and $B$ corresponding to optimum values of the reduced chi-squared, $\chi_{i}^{2}$, for the whole data set and for various subsamples (e.g. sorted by radio or X-ray class).

We obtained values of $\chi_{i}^{2}>5$ (very low significance) for most data selections including all those based on radio-selected AGN. The 10 radio-selected starbursts hosting X-ray-selected type-II AGN gave the most significant result, with $\chi_{i}^{2} \sim 3$

$\log L_{X}=(0.6 \pm 0.1) L_{R}+(21.4 \mp 2.4)$ 


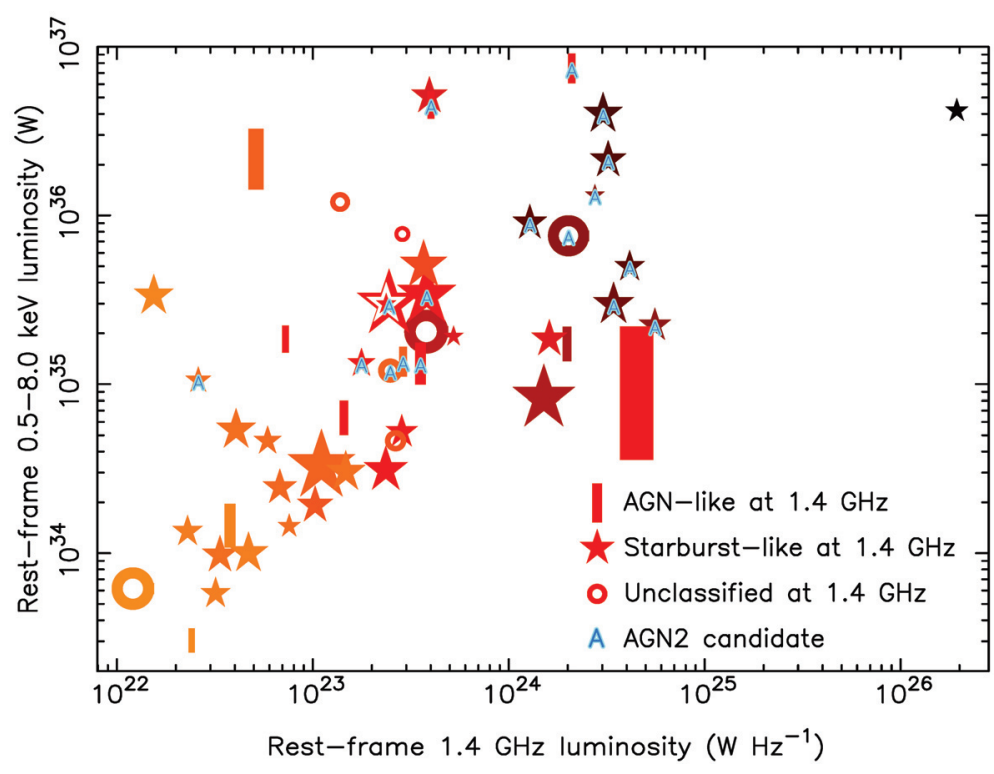

Fig. 4. The distribution of the classes of radio-bright sources (see key) with respect to $L_{\mathrm{R}}$ and $L_{\mathrm{X}}$. The shade of red is proportional to redshift. The paler, brighter and darker symbols represent the approximate redshift ranges $(z<1),(1<z<2)$ and $(z>2)$, respectively. The size of the symbols is proportional to the source largest angular size. The blue "A"s represent X-ray selected type-II AGN; the highest redshift source may also be in this category. Three sources marked as starbursts also contain radio AGN (not shown to avoid overcomplicating this plot), see Fig. 6. Sources not detected at $1.4 \mathrm{GHz}$ are not shown as they have no radio angular size measurements. (This figure is available in color in electronic form.)

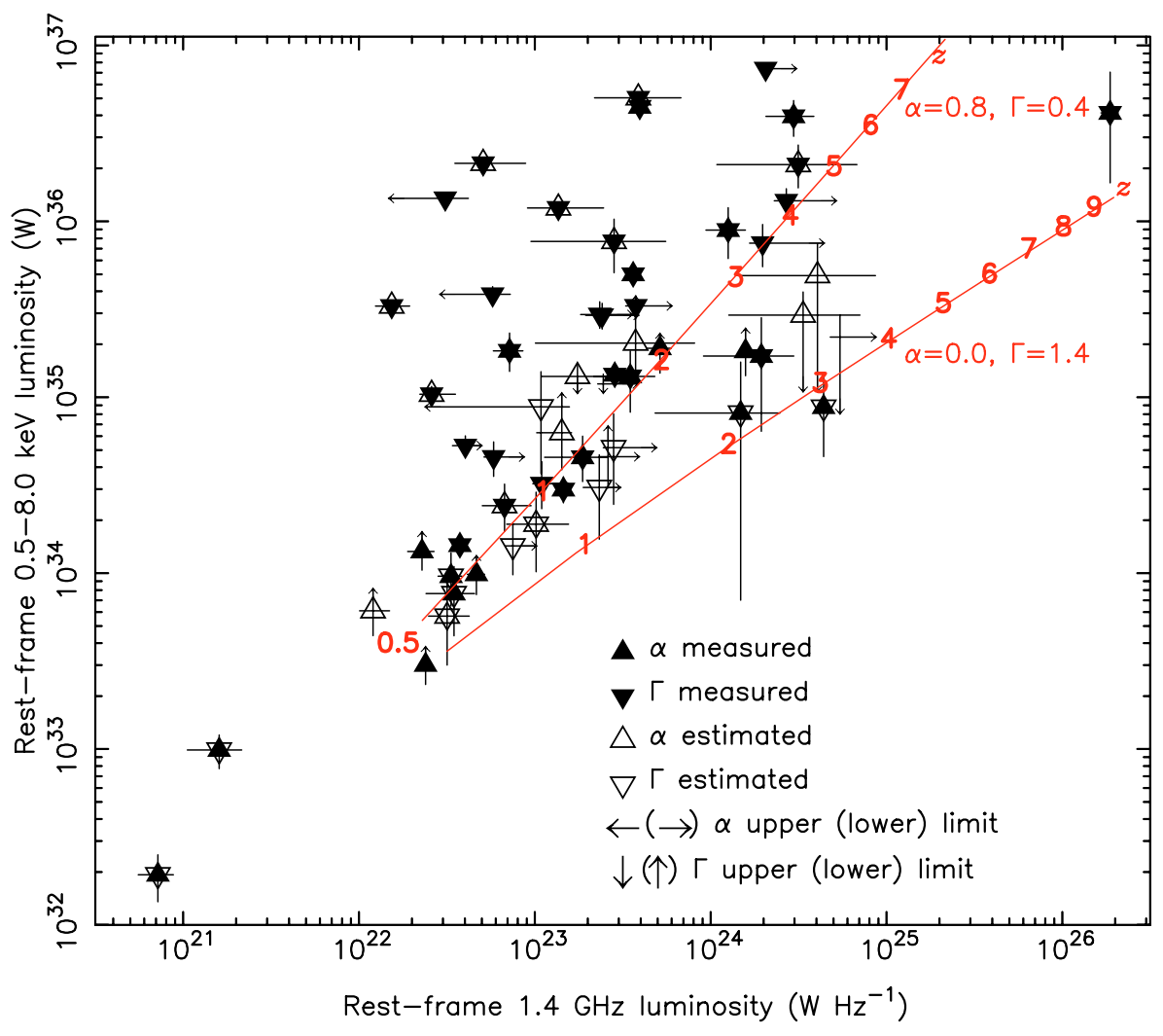

Fig. 5. The accuracy of $L_{\mathrm{R}}$ and $L_{\mathrm{X}}$ measurements. Uncertainties due to the spectral or photon index measurements are distinguished as shown in the key. See text for further explanations. The red lines show the detection limits at various redshifts for sources with the given combinations of $\alpha$ and $\Gamma$. For example, a source with $L_{\mathrm{R}}=10^{24} \mathrm{~W} \mathrm{~m}^{-2}$ is detectable at $z<2$ if it has $\alpha \sim 0$, but would be seen out to $z \sim 2.5$ if it had a steep radio spectrum. Its X-ray counterpart would be detected at $z \sim 2$ if it had $L_{\mathrm{X}}>5 \times 10^{34}$ and $\Gamma>1.4$, but would need to have $L_{\mathrm{X}}>10^{35}$ if it had $\Gamma<0.4$. (This figure is available in color in electronic form.) (the uncertainties show the range in which the minimum $\chi^{2}$ was obtained), shown by the purple line in Fig. 6. Including $\mathrm{J} 123642+621331$ as a radio starburst plus AGN (Sect. 5.3) gives an almost identical relationship, consistent with the suggestion that it may also host a type-II AGN, see Sect. 5.2. In any case, Tables 1 and 2 show that the lower error bound to $\Gamma$ gives $\mathrm{J} 123642+621331$ a flat enough photon index to be a type-II AGN and the corresponding value of $L_{\mathrm{X}}$ still fits within the uncertainties of Eq. (16).

We obtained $3<\chi_{i}^{2}<5$ for low-luminosity, low-redshift starbursts, e.g. $z<1.3, L_{\mathrm{X}}<10^{35} \mathrm{~W}$ (17 sources) gives

$\log L_{\mathrm{X}}=(0.95 \pm 0.05) \log L_{\mathrm{R}}+12.6 \mp 1.2$ shown by the green line in Fig. 6 . These coefficients are similar to those of Eq. (1) (Bauer et al. 2002b), shown by the red line in Fig. 6. The slight systematic offset to lower X-ray luminosities in our sample is probably because we used the $2 \mathrm{Ms}$ data and a default of $\Gamma=1.4$ where it was not measured. The low-redshiftdominated sample of Bauer et al. (2002b) has a higher average measured photon index and they took $\Gamma=2$ for sources which did not have a measured value in the $1 \mathrm{Ms}$ data. Six objects are common to both samples.

Barger et al. (2007) dispute the existence of the radio-X-ray luminosity relationship, as applied to high-redshift samples including the $\operatorname{HDF}(\mathrm{N})$, suggesting that it is a selection effect. We cannot compare this directly with our weak correlation given in 


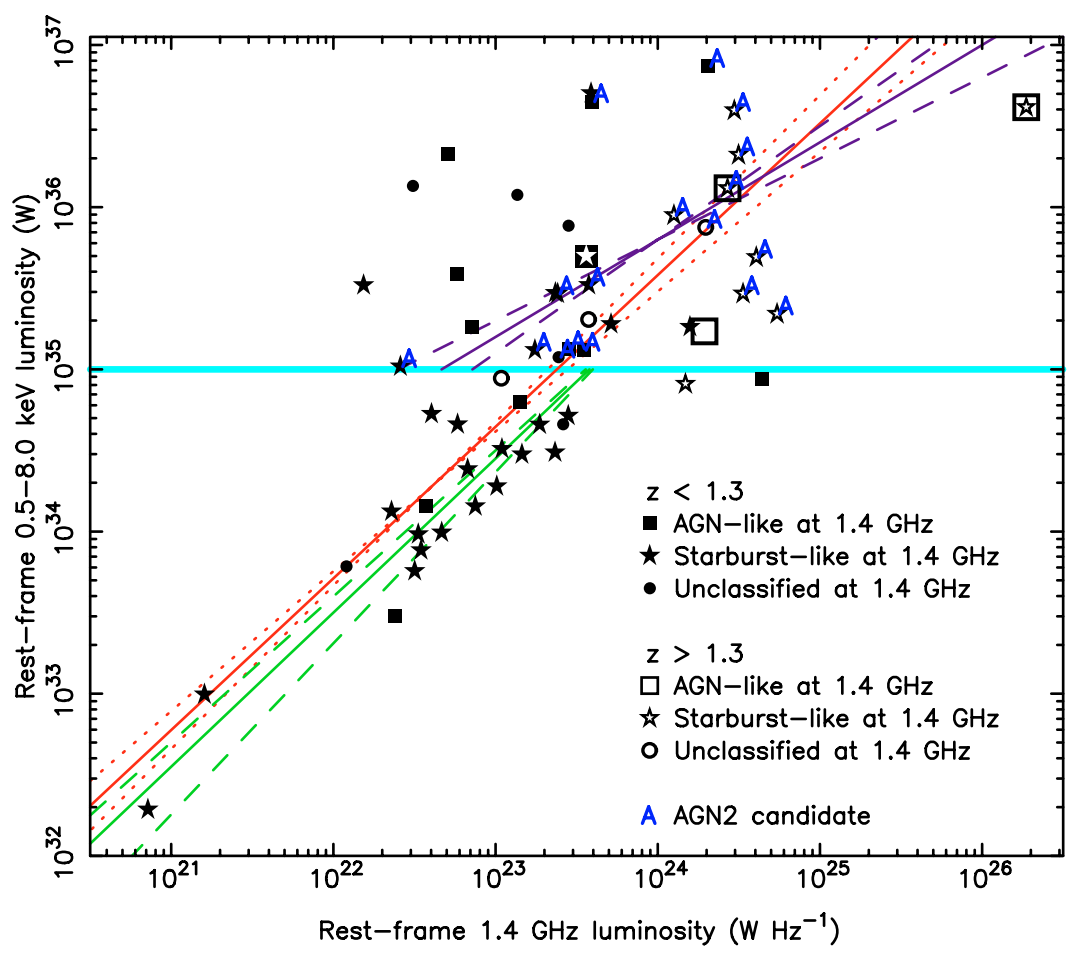

Fig. 6. X-ray luminosity as a function of radio luminosity for all 57 radio sources with X-ray counterparts and measured redshifts, classified as shown in the key (the squares enclosing stars are the three starbursts with apparent radio AGN cores; the lowest luminosity one is at $z<1.3$ ). The X-ray luminosity limit for AGN, $L_{\mathrm{X}} \approx$ $10^{35} \mathrm{~W}$, is marked by a horizontal blue dividing line. The long red line is the relationship found by Bauer et al. (2002b) (Eq. (1)). The short green line shows the relationships for radio starbursts at $z<1.3$ with $L_{\mathrm{X}}<10^{35} \mathrm{~W}$ (Eq. (17)). The short purple line shows the relationship for radio starbursts hosting X-ray selected type-II AGN (Eq. (16)) at $L_{\mathrm{X}}>10^{35} \mathrm{~W}$. The dashed lines show the uncertainties for each relationship. The blue As indicate X-ray selected type-II AGN; the most radio-luminous sources may also be in this category. (This figure is available in color in electronic form.)
Eqs. (16) and (17) since about half of our radio starbursts with obscured X-ray AGN and a third of low-X-ray luminosity starbursts have $S_{\mathrm{R}}$ less than their cutoff of $60 \mu \mathrm{Jy}$, and our criteria for starburst classification is more specific to the origins of the radio emission than is their optical method. The significant point for both this paper and Barger et al. (2007) is that although a high proportion of high-redshift star-forming sources detected in the radio are also detected in X-rays, their luminosities are weakly correlated or uncorrelated, suggesting that the X-ray emission is of non-starburst origin.

Figure 6 also shows that 2 out of 4 radio AGN with $L_{\mathrm{X}}<10^{35} \mathrm{~W}$ lie close to the starburst-based relationships and the other 2 are under-luminous in X-rays (although one of these, the source with the lowest value of $L_{X} / L_{R}$, is the FR 1 $\mathrm{J} 123644+621133$, the only radio-X-ray crossmatched source to have jet-dominated radio emission). Conversely, only one each of a radio starburst and an unclassified source are present with $z>1.3$ and $L_{\mathrm{X}}<10^{35} \mathrm{~W}$ although as shown by Fig. 5, such a source detected in one regime would also be detected in the other out to $z \sim 2$ for a typical starburst spectral index.

We emphasise that the significance of quantitative luminosity relationships is low for samples drawn exclusively from the $\mathrm{HDF}(\mathrm{N})$ data. The presence of $\mathrm{X}$-ray and radio emission appears to be correlated (Sect. 2.5), but not the precise luminosities, whether starbursts or any other classes of objects are studied. The clear correlation between $L_{X}$ and $L_{R}$ found for samples dominated by lower-redshift sources does not apply to the high-redshift, high-luminosity sources. We already noted (in Sect. 2.5) that the nature of the overlap between radio and X-ray detections changes around $z \sim 1.1$, which supports our contention that the relationship between radio- and X-ray-emission mechanisms changes dramatically around $z=1.1-1.3$.

\subsection{Hard-band X-ray luminosity}

The values of $L_{X}$ given in Table 2, derived using Eq. (11) in Sect. 4.2, do not take into account corrections for absorption nor for the intrinsic photon index. Padovani et al. (2004) derived the rest-frame X-ray luminosities for candidate type-2 AGN using the observed hard-band flux densities and assuming an intrinsic $\Gamma=1.8$. We follow this method to calculate $L_{\mathrm{XH}}$ for all the crossmatched sources with published redshifts, shown as the arrow ends in Fig. 7. We looked for relationships between $L_{\mathrm{XH}}$ and $L_{\mathrm{R}}$ using a method similar to that described in Sect. 6.1 but btained results of even lower significance.

Figure 7 shows that all the type- 2 AGN have $L_{\mathrm{XH}}>L_{\mathrm{X}}$, as expected, and that the difference is greater for the more radioluminous sources. All sources with arrow tips above the blue horizontal line have $L_{\mathrm{XH}}>10^{35} \mathrm{~W}$, indicating the presence of an X-ray AGN (Alexander et al. 2003a; Cowie et al. 2004a).

We used Eq. (1) to predict the full-band X-ray luminosity of starburst origin, $L_{\mathrm{XSB}}$, assuming that $L_{\mathrm{R}}$ was entirely due to starburst activity. $L_{\mathrm{XSB}}$ is an overestimate where a significant fraction of radio emission is of AGN origin, although $\mathrm{J} 123642+621331$ is the only powerful starburst where over half the radio emission is AGN-powered (Sect. 5.1). As it has been shown empirically (e.g. Ranalli et al. 2003) that hard- and softband X-ray emission of starburst origin have similar dependencies in the relationships with emission of common origin in other regimes, the difference between the measured hardband luminosity and $L_{\mathrm{XSB}}$ can thus be regarded as a lower limit to the X-ray luminosity of non-starburst origin. We find that $\left(L_{\mathrm{XH}}-L_{\mathrm{XSB}}\right)$ exceeds $10^{35} \mathrm{~W}$ for all 17 type-2 AGN identified by Padovani et al. (2004), including the 10 radio starbursts, with a mean difference of $z 3.9 \times 10^{36} \mathrm{~W}$ (much greater than the total uncertainties) for either sub-sample. We interpret this as confirming that these galaxies must contain AGN, since the excess $\mathrm{X}$-ray power, $\gg 10^{35} \mathrm{~W}$, is very unlikely to be be explained by any other mechanism. These values also suggest that, on average, $\lesssim 1 / 3$ of X-ray emission from obscured type-2 AGN is of starburst origin. All the radio starbursts with type- 2 AGN remain sufficiently X-ray-bright to meet the AGN selection criteria, even using the lower luminosity limit derived here. 


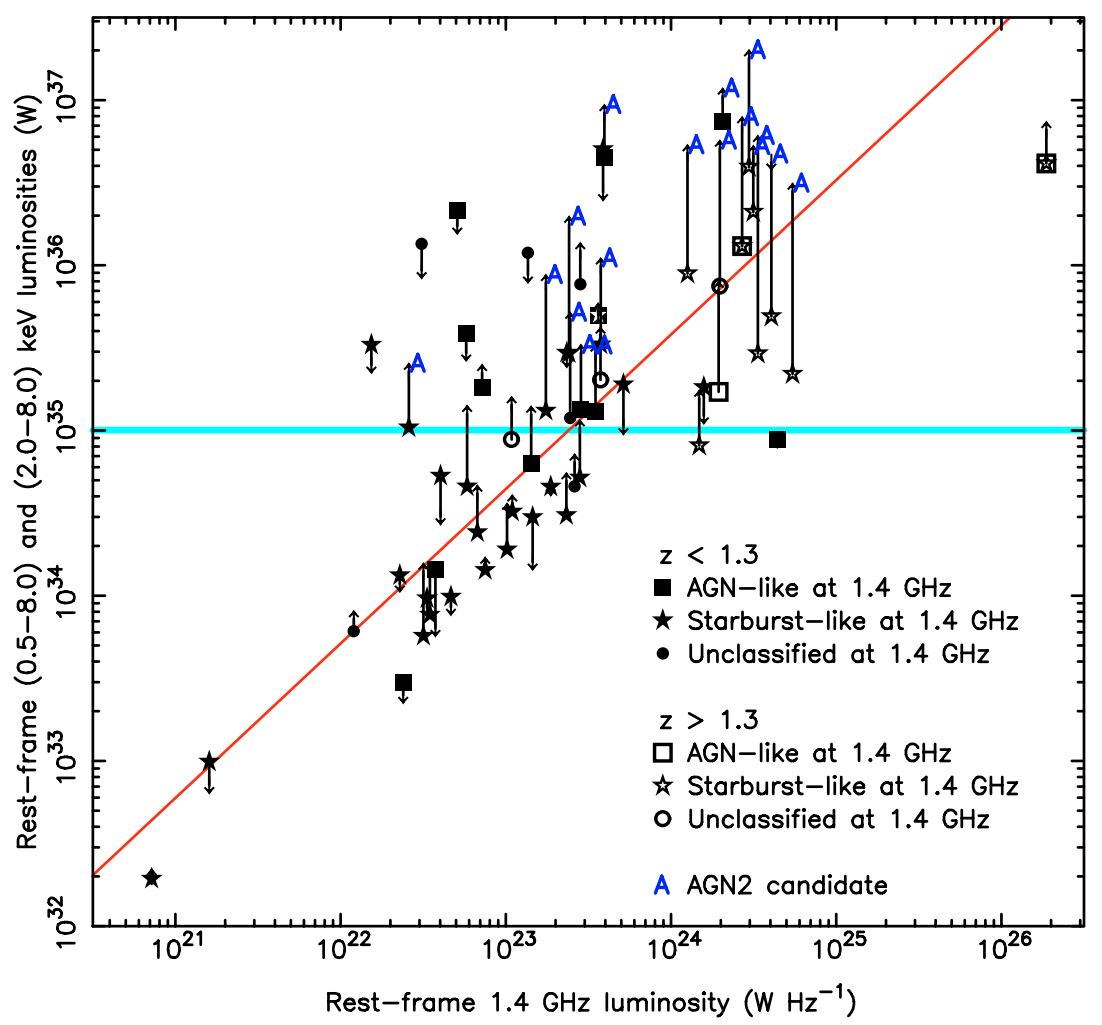

Fig. 7. The symbols, dividing line at $L_{\mathrm{X}} \approx 10^{35} \mathrm{~W}$ and red line are as in Fig. 6. The arrow tips show the $\mathrm{X}$-ray luminosity recalculated for the $2-8 \mathrm{keV}$ restframe, $L_{\mathrm{XH}}$, derived from the hard band flux densities using a constant $\Gamma=1.8$ in Eq. (11). The X-ray selected type-2 AGN are labelled at the $L_{\mathrm{XH}}$ values only. (This figure is available in color in electronic form.)
$L_{\mathrm{XH}}-L_{\mathrm{XSB}}$ is lower, at $\approx 1.6 \times 10^{36} \mathrm{~W}$ and $\approx 2.8 \times 10^{34} \mathrm{~W}$ for all radio-selected AGN and all starbursts respectively. Starbursts without type-II AGN show a very large scatter in the difference, about a mean of $\approx-2.1 \times 10^{36} \mathrm{~W}$, showing that any AGN contribution is negligible in the majority of "pure" starbursts.

\section{X-ray-selected type-II AGN associated with radio-selected starbursts}

\subsection{A distinct population}

Radio starbursts hosting type-2 AGN dominate the radio detections at high redshift, illustrated in Fig. 2. The 10-arcmin field contains 64 X-ray selected type-II AGN (as defined in Sect. 5.2), plus the candidate type-II AGN J123642+621331 (Sects. 5.3 and 6.1). Eighteen of these have radio-bright counterparts (none are among the additional $78.4-\mathrm{GHz}$ selected sources). $18 / 64$ is a similar proportion to the quarter of all X-ray sources in the 10arcmin field which are type-II AGN candidates. Nine out of the 11 radio-bright $\mathrm{X}$-ray sources at $z>1.3$ host type-II AGN and this includes 8 out of the 9 radio starbursts in this redshift range.

Figure 4 and Table 1 show that the majority of the X-ray selected type-II AGN with radio counterparts are associated with starbursts (classified using the criteria in Sect. 5.1). The breakdown by radio source type, redshift and luminosity is shown in more detail in Figs. 8 and 9. The 3 radio starbursts $(\mathrm{J} 123635+621424, \mathrm{~J} 123642+621331$ and $\mathrm{J} 123642+621545)$ which also show signs of containing radio AGN (Sect. 5.1) are only shown once, as starbursts. J123635+621424, and probably $\mathrm{J} 123642+621331$, are also X-ray selected type-II AGN.

Radio AGN outnumber starbursts 2:1 in the most powerful third $\left(S_{\mathrm{R}}>100 \mu \mathrm{Jy}\right)$ of all 92 radio-bright sources whilst starbursts are an even greater majority among the fainter sources. The radio-selected AGN are on average intrinsically more radioluminous than the starbursts. The median redshift of all radio AGN is higher compared with all starbursts in the $\operatorname{HDF}(N)$ and they appear to be separate populations (Muxlow et al. 2005). The high redshift radio-selected starbursts associated with obscured X-ray selected AGN appear to be a third class, distinct from the radio-bright AGN and from the majority of the starbursts.

Figure 10 shows a remarkable segregation between high- $\Gamma$, low- $z$, sources of all classes but without type-II AGN, and low- $\Gamma$ sources. Of the latter, the radio starbursts and unclassified sources with type-II AGN (including the candidate J123642+621331) show a slight correlation between $\Gamma$ and $z$, consistent with the change in X-ray spectral slope expected for highly obscured sources at higher rest-frame energies (Sect. 5.2). The blue $\Gamma(z)$ line is a very rough estimate of the change in the observed $\Gamma$ with increasing redshift, for a rest-frame $\Gamma=0$, based on the spectral models shown in Alexander et al. (2005a), their Fig. 7, for sources with $N_{\mathrm{H}} \gtrsim 2 \times 10^{27} \mathrm{~m}^{-2}$. All but 3 out of 16 sources with a $\Gamma$ below the estimated $\Gamma(z)$ line contain type-II AGN and the majority are radio starbursts; the only radio-bright AGN here is not a type-II AGN.

The 4 radio-bright AGN with X-ray type-II AGN all lie above this line, suggesting that they do not reach the highest degrees of obscuration. The segregation is not due to Comptonthick absorption as signs of this are only seen in one source, J123622+621629, a radio starburst hosting a type-II AGN, (Alexander et al. 2003b).

Although all but 2 of the radio AGN with measured redshifts are detected by Chandra, the non-detections are the highestredshift sources; in contrast, all the starbursts known to have $z>1.3$ have X-ray counterparts. Figures 8 and 9 (panels a and b) show that the distributions of X-ray selected type-II AGN are biased towards much higher redshifts and luminosities than the distributions of radio-classified AGN or unclassified radio sources. The population of type-II AGN does overlap closely the high redshift/high luminosity end of the distribution of radioselected starbursts (panel c). 

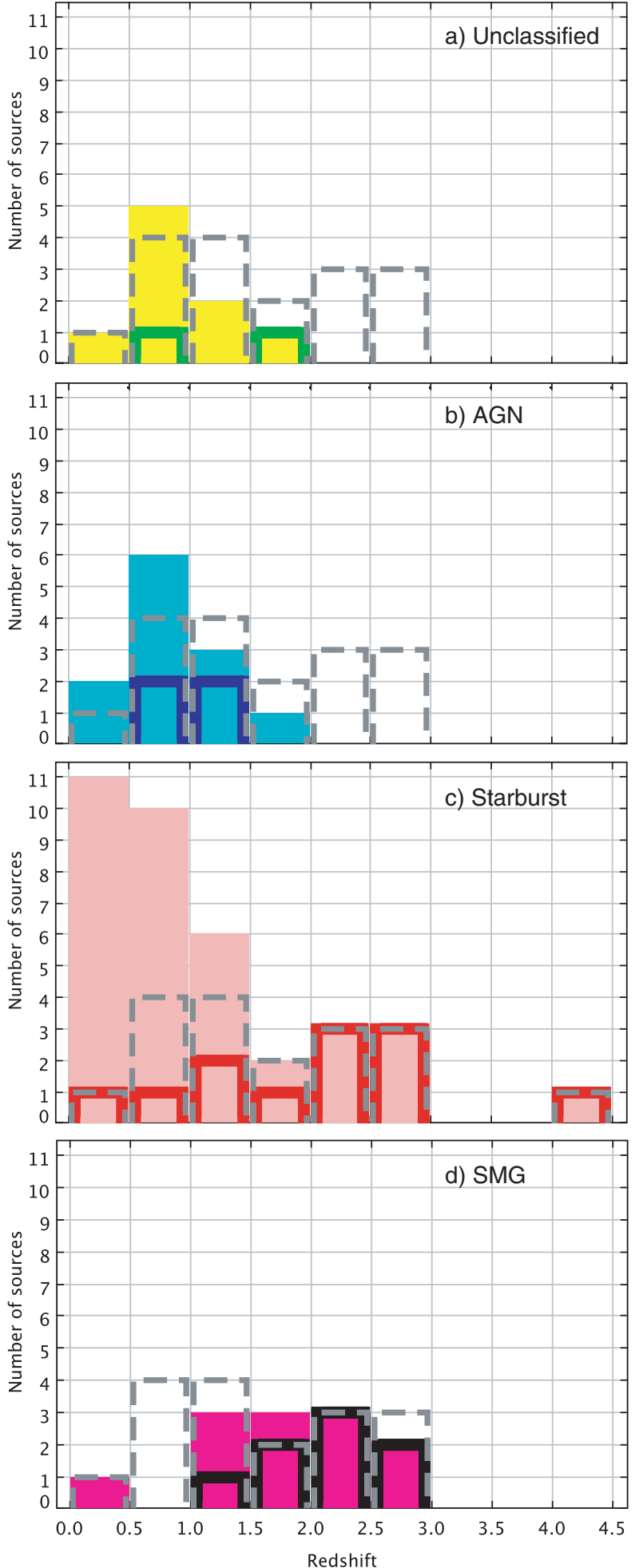

Fig. 8. Redshift distribution of radio/X-ray sources according to radio classification and counterparts. In each panel the dashed grey line shows the distribution of sources which have X-ray type-II AGN signatures (including J123642+121331, Sect. 5.3) and the solid line shows their counterparts in the sub-sample. The filled area shows the total number of sources with radio properties which are a) unclassified, b) AGNlike or c) starburst-like; panel d) shows radio sources with SCUBA counterparts. (This figure is available in color in electronic form.)

\subsection{Radio-bright SMG starbursts with type-II AGN}

There are strong similarities between the redshift distributions of the radio SMGs and type-II AGN (Figs. 8 and 9, panel d),
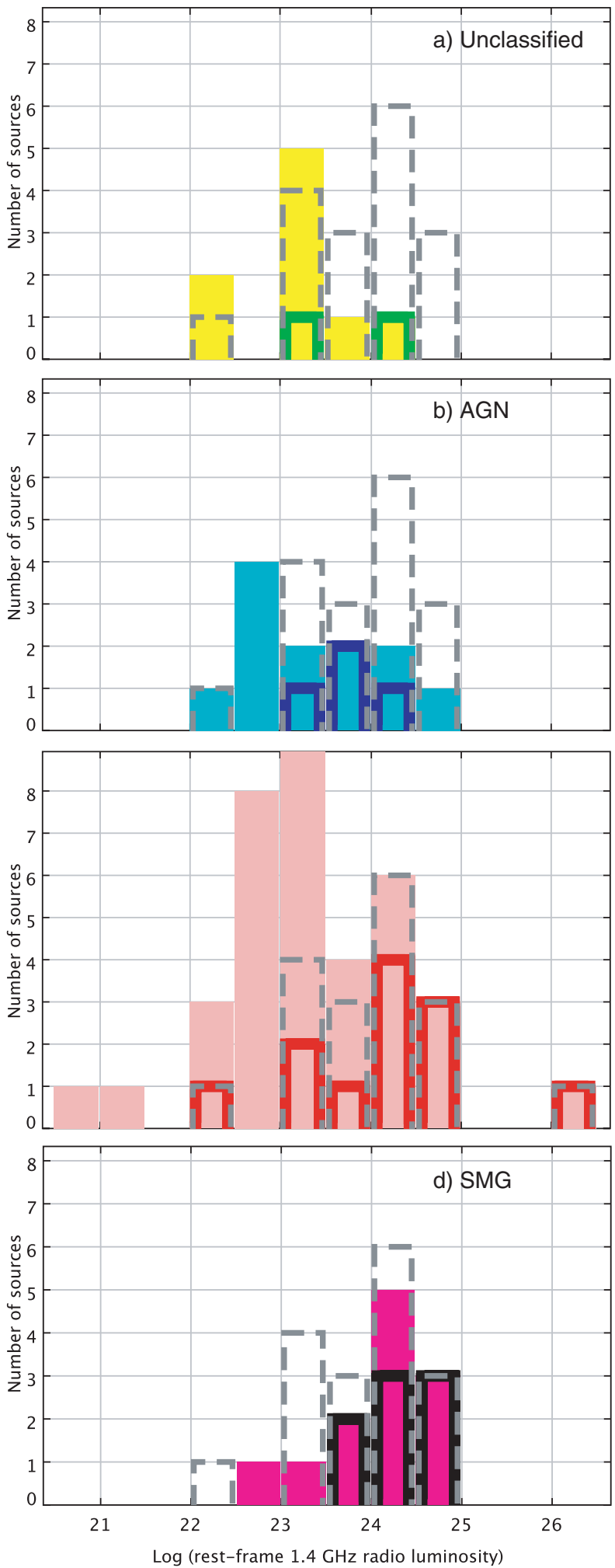

Fig. 9. Radio luminosity distribution of radio/X-ray sources according to radio classification and counterparts, see Fig. 8 for more details. (This figure is available in color in electronic form.)

especially at higher $z .20 \%$ of all radio-bright galaxies detected by Chandra are also SCUBA sources (Sect. 2.4.2) and nearly half of these contain type-II AGN. We noted a low correlation between $L_{X}$ and $L_{R}$ for high-redshift starbursts (Sect. 6.1); a similar large scatter was seen by Borys et al. (2004) for a SCUBAselected subsample of 10 sources. 
Alexander et al. (2005a) calculated the radio and X-ray luminosities for all SMG with spectroscopic redshifts, in the overlap between the Chandra and VLA fields of view. All except one have X-ray luminosities in excess of the relationship predicted from star formation, by over an order of magnitude in the case of the type-II AGN candidates (as we also found in Sect. 6.2). The $\mathrm{X}$-ray selected type-II AGN also have an FIR/X-ray luminosity ratio about an order of magnitude greater than the typical ratio for nearby QSO, showing that at least $90 \%$ of the FIR emission is probably of starburst origin, at an SFR of order $1000 M_{\odot} \mathrm{yr}^{-1}$.

\subsection{Extended radio emission, compact $X$-ray cores}

The starburst interpretation of sub-mm emission from SMG which are hard X-ray sources is only questionable if they possess nuclear dust to much greater optical depths than is seen around local AGN (Sect. 1; Alexander et al. 2005a). We summarise the evidence that these objects do contain extended starbursts which are distinct from any AGN cores.

The radio starburst classification is based on distinctive morphology and spectral index (Sect. 5.1; Muxlow et al. 2005). The median angular size of radio-bright AGN with X-ray counterparts is $\sim 0$ '. 6 whilst for starbursts it is $\sim 11^{\prime \prime} 4$ (Table 1; Fig. 4). SCUBA galaxies which are most extended in the radio are also more likely to be X-ray bright; of the 12 SMG radio sources studied by Chapman et al. (2004b), the 6 with the largest 1.4-GHz angular sizes all had X-ray counterparts but only 2 of the 4 smallest radio sources had X-ray counterparts.

$\mathrm{X}$-ray emission from many of the the starburst galaxies would, however, be resolved, if it had a common origin with the radio emission, which is not seen - all $55 \mathrm{X}$-ray sources are smaller than the $1^{\prime \prime}$ Chandra FWHM in this region.

Nine out of the 10 radio-bright X-ray sources which are also SMG are radio starbursts with $\alpha \geq 0.7$ (including 7 with type-II AGN) and the tenth is unclassified. The mean radio angular size is 1 .'3 and only $\mathrm{J} 123635+621424$, a starburst with a radio AGN core, is smaller than 0.'6. Chapman et al. (2004b) found a similar result, obtaining a typical extent of 1 " for the radio counterparts to a sample of dozen SMGs. Pope et al. (2005) found that the the optical ACS counterparts of SMG at $z<2$ in the HDF(N) have radii in the range $\sim 1^{\prime \prime}-\sim 22^{\prime \prime} 5$, which is significantly larger than field galaxies at the same redshift. Similarly, more distant $(z \approx 2.5)$, highly reddened galaxies with SEDs consistent with vigorous star formation are typically over twice the size of nonstarformers in HST NICMOS images of the HDF(S) (Zirm et al. 2007).

We conclude that, although present instruments cannot resolve MIR or sub-mm sources on the scale of radio or optical emission with starburst characteristics, the close association supports a common origin. In contrast, X-ray emission from the same sources is consistently more compact than the radio emission and appears to have a distinctly different origin.

\section{The nature of very faint radio and X-ray sources}

At high redshifts, the present generation of radio and X-ray surveys are biased towards extreme objects, as whatever was "normal" at $z \gg 1$ is below the threshold for secure detections of individual sources. In advance of $e$-MERLIN, the EVLA and XEUS, stacking is the only method to investigate the radio and X-ray properties of objects at $z>1$ with $L_{\mathrm{R}}<10^{23} \mathrm{~W} \mathrm{~Hz}^{-1}$ or $L_{\mathrm{X}}<10^{34}$ W. Muxlow et al. (2005) demonstrated the existence of such a radio population by finding a statistical excess of $1-6 \sigma$

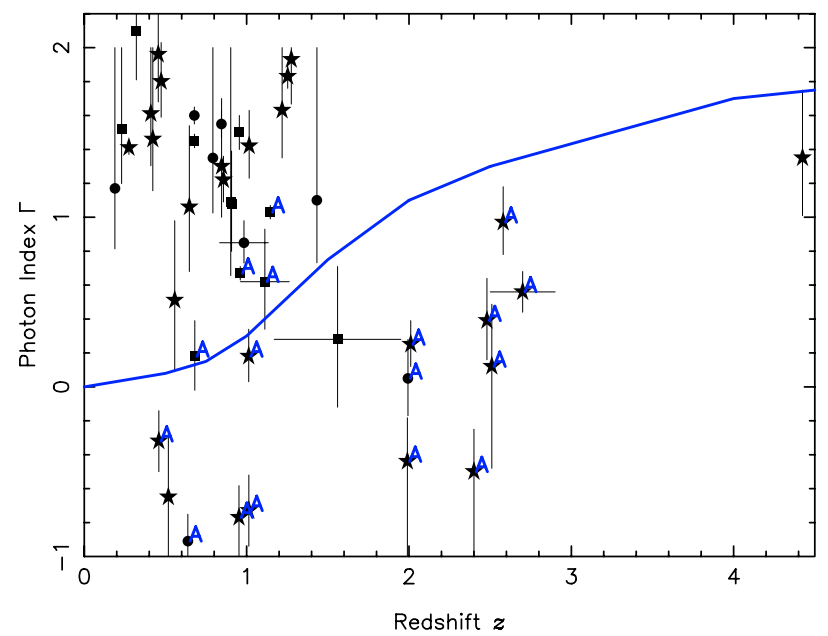

Fig. 10. X-ray photon index as a function of redshift for radio starbursts (stars), AGN (squares) and unclassified sources (circles). All photon indices are fully measured or are partly measured upper or lower limits (see Sect. 4.2). X-ray selected type-II AGN are labelled A. The blue line shows a rough extrapolation of an observed-frame photon index $\Gamma=0$ at $z=0$ out to $z=4.5$, using estimates based on the models shown in Alexander et al. (2005a) for sources with $N_{\mathrm{H}} \geq 2 \times 10^{27} \mathrm{~m}^{-2}$. (This figure is available in color in electronic form.)

radio flux on arcsec scales at the positions of ISO sources and of optical (HST WFPC2) galaxies with $I \lesssim 24 \mathrm{mag}$ in the 3 -arcmin field. The MERLIN+VLA images are more than twice as sensitive as the VLA-only data but no new sources were detected in the 3-arcmin field between $27-40 \mu \mathrm{Jy}$, suggesting that any objects just below the VLA completeness limit are extended over $\gtrsim 2^{\prime \prime}$ with no hotspots. Garrett et al. (2000) showed that about $10 \%$ of radio sources in the $\mathrm{HDF}(\mathrm{N})$ are probably resolved out even at $2^{\prime \prime}$ resolution so even stacking MERLIN+VLA data will not recover these.

We analyse the excess radio flux at the positions of Chandra sources in the recently-made 8 -arcmin radio image convolved with a restoring beam of 0.4 (see Sect. 3.2). Muxlow et al. (2006) and Beswick et al. (2006) present similar analyses at the positions of ACS and Spitzer sources.

\subsection{Radio-faint Chandra sources}

181 X-ray sources from the catalogue of Alexander et al. (2003b) lie within the 8 -arcmin MERLIN+VLA 1.4-GHz image. We used the matching criteria described in Sect. 2.2 to divide the $\mathrm{X}$-ray sources into 39 sources with radio counterparts brighter than $40 \mu \mathrm{Jy}$ ("radio-bright"), and 142 without ("radio-faint"). For each sample, we stacked the 1.4-GHz radio flux density enclosed by 0.25 to $2^{\prime \prime}$ radius circles centred on the X-ray source positions. We constructed control samples by stacking the radio flux at positions offset by $10^{\prime \prime}$ from each X-ray source (checking that these did not coincide with known X-ray or radio sources or their sidelobes). The noise distribution is slightly non-Gaussian due to confusing sources in the sidelobes of the heterogeneous primary beams but there are no artifacts $>7 \sigma(\approx 27 \mu \mathrm{Jy}$ per $0 . ' 2-0 . ' 5$ beam) apart from sidelobes very close to the 3 sources brighter than $1 \mathrm{mJy}, \mathrm{J} 123644+621133, \mathrm{~J} 123714+620823$ and J123725+621128 (Muxlow et al. 2005). The average stacked flux density from the radio-faint sources increased up for radii up to $\sim 1^{\prime \prime}$ and levelled off for higher radii, so we report results for $1^{\prime \prime}$ radii. The variance in each bin is $3-4 \mu \mathrm{Jy}$ (the radio map $1 \sigma$ level) and the control samples for the radio-faint sources are all $<3 \sigma$. 


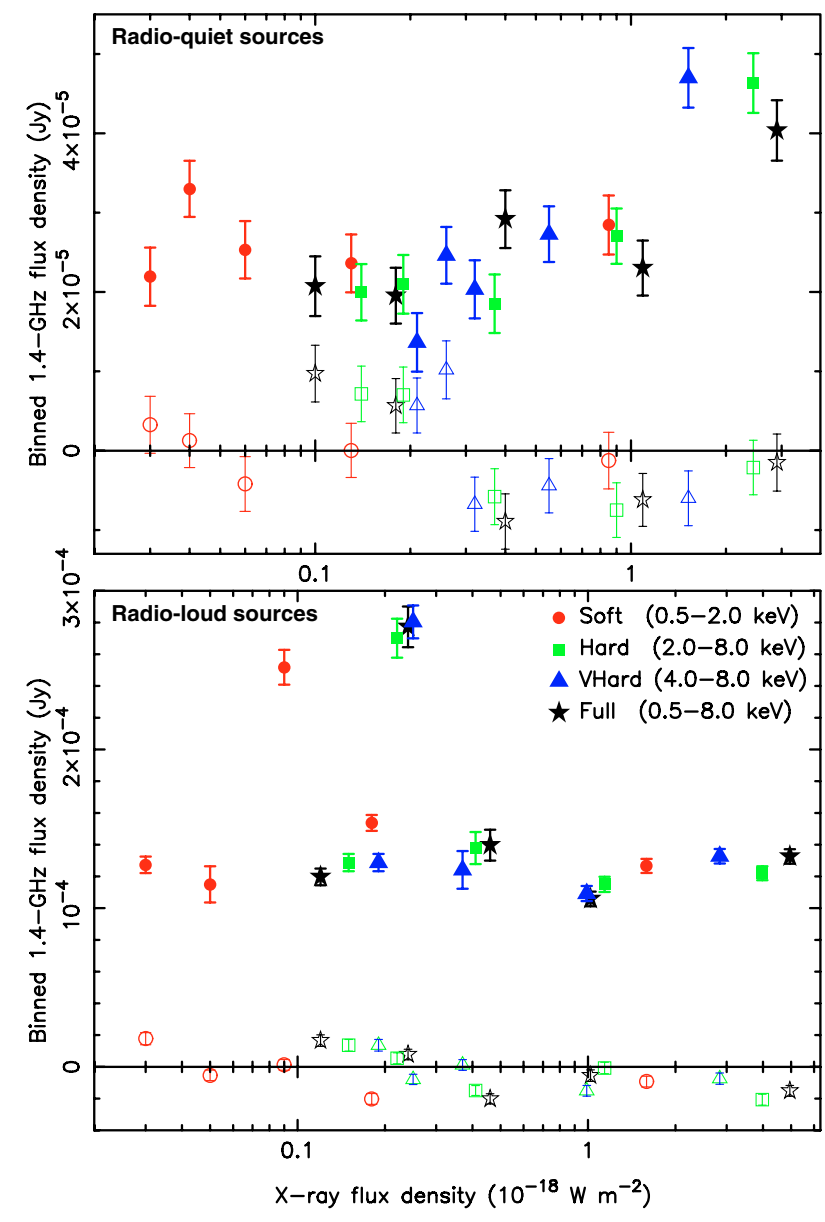

Fig. 11. The heavy symbols in the upper and lower panes show the binned 1.4-GHz flux densities at the positions of radio-faint and -bright $\mathrm{X}$-ray sources, respectively. This was measured separately for $4 \mathrm{X}$-ray bands, see key. The ordinate axis shows the median X-ray flux in each bin for each band. The faint hollow symbols show the binned $1.4-\mathrm{GHz}$ flux densities for control samples at $10^{\prime \prime}$ separation from each X-ray source. The error bars show the rms scatter in each bin. (This figure is available in color in electronic form.)

The stacking intervals were sorted by X-ray flux density, photon index and redshift, into bins containing almost equal numbers of measurements. In each of Figs. 11 and 12 the scale on the abscissa shows the median value in each bin.

\subsubsection{Stacking by X-ray flux density}

We present results for stacking sorted by full, soft, hard and very hard (4-8 keV) band flux density. We plot the relationship between the mean stacked radio flux density and the X-ray flux density in each of 5 bins with 28 or 29 (7 or 8) samples in each bin for the radio-faint (-bright) sources. We treated upper limits as values; hence the nominal value for the bottom bin may be greater than the true median X-ray flux but it does not affect the significance of the results. The filled symbols in Fig. 11, upper panel, show the stacked radio flux at the X-ray positions of radio-faint sources (see key) and the hollow symbols show the corresponding control values. This shows that the mean stacked radio flux density is significantly above the control for all bins apart from the faintest very hard band bin. The average radio flux density is similar for any soft-band X-ray flux density, but becomes increasingly correlated with X-ray flux density for the hard and very hard bands. Figure 11, lower panel, shows that

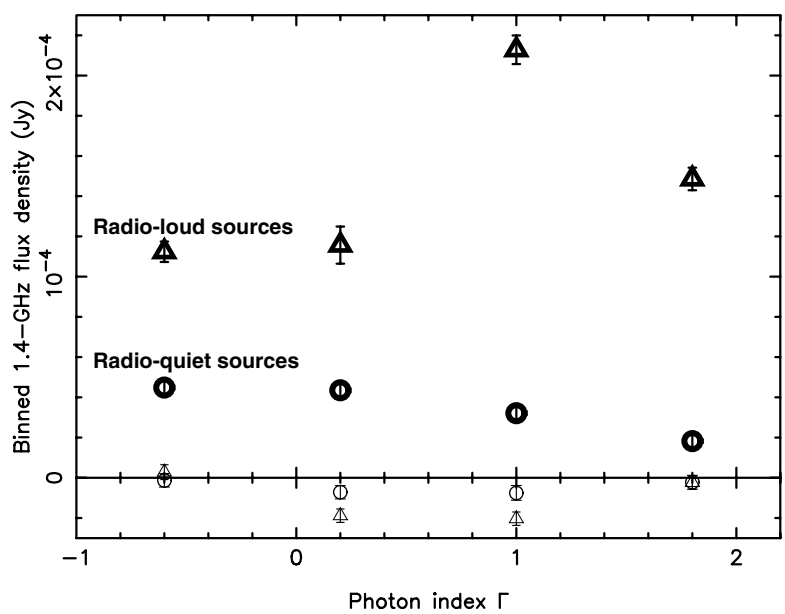

Fig. 12. The heavy circles and triangles show the binned $1.4-\mathrm{GHz}$ flux densities at the positions of radio-faint and -bright X-ray sources, respectively. The ordinate axis shows the median X-ray photon index in each bin for each band. The faint hollow symbols show the binned 1.4-GHz flux densities for control samples at $10^{\prime \prime}$ separation from each $\mathrm{X}$-ray source. The error bars show the rms scatter in each bin.

there is no correlation with any band for radio-bright sources (the four high values are biased by one very bright radio source), as discussed in Sect. 6.

\subsubsection{Stacking by photon index}

84 (29) radio-faint (-bright) X-ray sources have at least partly measured photon indices $(\Gamma)$ (i.e. detected in at least one subband as well as full band). We plot the mean stacked radio flux density against the X-ray photon index in each of 4 bins evenly spaced in $\Gamma$, which gives similar source counts in each bin. Figure 12 shows that radio-faint $X$-ray sources (heavy circles) with $\Gamma<0.6$ have a mean radio flux density twice that for sources with $\Gamma>1.4$. This is not the case for the radio-bright sources (heavy triangles).

\subsubsection{Stacking by redshift}

76 (35) radio-faint (-bright) X-ray sources have measured redshifts. In each case we divided them into 4 bins with approximately equal numbers of samples in each bin. There is no obvious correlation for either sample.

\subsection{The nature of radio-faint $X$-ray sources}

There is a significant excess of radio flux at the positions of Chandra sources without formally identified radio counterparts. These have typical $1.4-\mathrm{GHz}$ flux densities from $\approx 20-40 \mu \mathrm{Jy}$ per $1^{\prime \prime}$-radius circle $\left(\equiv 6-13 \mu \mathrm{Jy} \operatorname{arcsec}^{-2}\right.$ ). This is the median largest angular size of radio sources above $40 \mu \mathrm{Jy}$ (Muxlow et al. 2005) and is three times the median Chandra position error for the sources within the 8-arcmin field (Sect. 2.2).

The most obvious correlations for radio-quiet sources are the association of a higher average radio flux density with a higher X-ray flux density in the full or harder bands, and with a lower $\Gamma$. The average radio flux density approaches $40 \mu \mathrm{Jy}$ for radioquiet X-ray sources with flux densities $>10^{-18} \mathrm{~W} \mathrm{~m}^{-2} \mathrm{~Hz}^{-1}$ or with hard photon indices, suggesting that the majority of these (probably obscured) X-ray sources have genuine radio counterparts. We examined the radio-quiet images at the position of 
each Chandra source by plotting contours at 3, 4, 5 and $6 \sigma^{13}$ Eleven sources are $>20 \mu \mathrm{Jy}(5 \sigma)$. Their apparently extended radio flux and the absence of hotspots brighter than $27 \mu \mathrm{Jy}$ per 0.4 beam, corresponding to maximum brightness temperatures of less than a few $100 \mathrm{~K}$, suggests that these sources are part of the radio starburst population and do not contain radio-bright AGN. Seven of the corresponding X-ray objects have a measured photon index of which 5 have $\Gamma<1$ and 4 are listed as type-II AGN by Padovani et al. (2004). A similar proportion (40\%) of all 142 radio-faint Chandra sources have low, measured photon indices characteristic of obscured AGN.

The stacked-average photon index for the whole Chandra $\mathrm{HDF}(\mathrm{N})$ field becomes flatter for lower X-ray count rates and then slightly steepens again (Alexander et al. 2003b). The composite X-ray spectra of the most obscured Chandra sources $\left(N_{\mathrm{H}}>5 \times 10^{27} \mathrm{~m}^{-2}\right)$ show an upturn at rest frame energies $<4 \mathrm{keV}$, attributed to star formation (Alexander et al. 2005a). This is independent evidence for the association between obscured AGN and starburst activity.

$\mathrm{X}$-ray sources associated with individual galaxies in the 8 -arcmin field are smaller than the $\approx 1$ " resolution of Chandra, and none are among the extended emission sources identified by Bauer et al. (2002a). About $20 \%$ (29/142) of the radio-faint $\mathrm{X}$-ray sources have $F_{\mathrm{X}}>0.3 \times 10^{-18}$ and $\Gamma>1.4$. Equation (1) predicts a radio flux density $>40 \mu \mathrm{Jy}$ from such sources if the $\mathrm{X}$-ray emission is of starburst origin, so they would have been detected as radio-bright if the radio and X-ray emission had a common starburst origin. Instead, it is likely that these radioquiet sources contain unobscured X-ray AGN.

Almost all the remaining $40 \%$ of the radio-faint sources, with $F_{\mathrm{X}} \leq 0.3 \times 10^{-18}$, do not have measured photon indices (Bauer et al. 2004). Reddy \& Steidel (2004) stacked Chandra soft-band and VLA-only radio $\operatorname{HDF}(\mathrm{N})$ flux densities for (restframe) UV-selected objects at a mean redshift of $\sim 2$. Their measurements were consistent with the relationship found by Bauer et al. (2002b), giving an average SFR of $\sim 50 M_{\odot} \mathrm{yr}^{-1}$. Stacking Chandra data for Lyman Break galaxies out to $z=6$, gave similar results. Their selection criteria (optical detection, softband dominated) excluded objects like the SMGs and the most vigorous radio starbursts associated with type-II AGN, so these sources are probably analogues of the local ULIRGs.

In Sect. 2.5 we used the Kolmogorov-Smirnov test to show that there seemed to be a change in the relationship between radio and X-ray sources at redshifts above and below $\sim 1.1$. We also found (Sect. 6.1) a starburst-like relationship between $L_{R}$ and $L_{\mathrm{X}}$ for most low-redshift sources, but only very weak relationships at high redshift, most significant for the radio starbursts hosting type-II AGN. The correlation between radio-faint radio flux density and harder X-ray emission seen in Fig. 11 (upper) is consistent with a continuation of this relationship for the radiofaint sources. The correlation with harder photon index is also consistent with type-II AGN powering the the dominant population of distant X-ray sources with radio counterparts of a few tens of $\mu \mathrm{Jy}$.

In summary, we predict that future instruments will confirm that the association between the presence of X-ray and radio emission extends to radio flux densities $\lesssim 10 \mu \mathrm{Jy} \operatorname{arcsec}^{-2}$. The available evidence suggests that two fifths of these sources will be found to be radio starbursts hosting obscured X-ray AGN, probably at high redshifts, one fifth are radio-quiet, unobscured AGN and the remainder are probably ULIRGs.

\footnotetext{
13 An image cut-out service will be available via AstroGrid.
}

\section{Conclusions}

We have used Virtual Observatory tools and RadioNet software to compare the radio and X-ray properties of galaxies at $z \lesssim 4.5$ in the $\operatorname{HDF}(\mathrm{N}) .92$ radio-bright objects $\left(S_{\mathrm{R}}>40 \mu \mathrm{Jy}\right)$ are resolved by MERLIN+VLA at $1.4 \mathrm{GHz}$. 55 of these sources are also securely detected by Chandra and the majority of these sources have spectral/photon indices based on measurements at more than one frequency. Where spectral/photon indices are estimated, we have not used the values as primary diagnostics.

\subsection{The majority of radio starbursts in the $H D F(N)$ have $X$-ray counterparts}

The combination of radio morphologies and spectral indices, in some cases supported by rest-frame MIR measurements, provides reliable diagnostics for the origins of the radio emission from most of the sources (Sect. 5; Muxlow et al. 2005). 70\% of radio starbursts have X-ray counterparts. Radio starbursts outnumber radio AGN by 3:1 for classified sources in the whole radio-bright sample and the same proportion is seen for the 55 sources with X-ray counterparts. Seven unresolved $8.4-\mathrm{GHz}$ sources which are not radio-bright at $1.4 \mathrm{GHz}$ also have X-ray counterparts are not included in most of this analysis, but they appear to contain a similar majority of starbursts. In contrast, the X-ray emission is of AGN origin in about $80 \%$ of all Chandra sources (Bauer et al. 2002b).

Optical spectroscopy finds approximately equal numbers of star formers and AGN (Cowie et al. 2004a) and only 6 opticallyclassified starbursts in the $\operatorname{HDF}(\mathrm{N})$ have X-ray counterparts (along with 29 optical AGN). Similarly, only a few percent of the starbursts found using $B z K$ criteria, (Daddi et al. 2004), have soft-X-ray counterparts (Daddi et al. 2005) (hard-X-ray sources were excluded from their sample). These results imply that many radio starbursts which are too obscured to be identified using optical or even NIR spectroscopy and photometry, are a separate population with a much higher proportion of X-ray counterparts.

\subsection{High-luminosity radio and $X$-ray emission has separate origins with the same galaxies}

There is no discernible relationship between either the radio and X-ray flux densities or the $K$-corrected luminosities for the cross-matched sample as a whole. The close relationship between $L_{\mathrm{R}}$ and $L_{\mathrm{X}}$ established by Bauer et al. (2002b) breaks down at $z \gtrsim 1.3$, even if only radio starbursts are considered (Sect. 6.1). The X-ray emission predicted from the radio luminosity of these sources is on average less than $1 / 3$ of the observed $\mathrm{X}$-ray luminosity and even after subtracting the potential starburst contribution, the hard-band X-ray luminosity of the type-II AGN still exceeds $10^{35} \mathrm{~W}$ (Sect. 6.2). At least half of the radio sources with $\mathrm{X}$-ray counterparts have a largest angular size greater than the Chandra resolution of $\approx 1^{\prime \prime}$ but all the X-ray sources are unresolved.

The presence of detectable radio emission is significantly correlated with the probability of also detecting X-rays, and vice versa (Bauer et al. 2002b) but our results strongly suggest that the radio and $\mathrm{X}$-ray emission originates from separate phenomena in most sources at $z \gtrsim 1.1$. 


\subsection{A distinct population of high-redshift radio/sub-mm starbursts associated with X-ray-selected obscured AGN}

The fraction of X-ray sources with radio counterparts increases significantly at $z \gtrsim 1.1$ (Fig. 2). Section 7 sums up the evidence that high- $z$ radio- or sub-mm-selected starbursts are a separate population from starforming galaxies at $z \lesssim 1.3$ and indeed exceed the activity seen in local ULIRGs (e.g. Sect. 2.4.2, and references therein.)

A hard photon index $\Gamma \leq 1$ (at rest-frame $0.5-8 \mathrm{keV}$ ) combined with $L_{X}>10^{35} \mathrm{~W}$ shows the presence of an obscured typeII AGN and 18 such objects identified by Padovani et al. (2004) have radio counterparts in the $\operatorname{HDF}(\mathrm{N})$. Model X-ray spectra extending to high rest-frame energies (Alexander et al. 2005a) suggest that type-II AGN at $z \gtrsim 2$ could have slightly higher $\Gamma$ as measured by Chandra. This is supported by the $L_{\mathrm{R}}-L_{\mathrm{X}}$ relationship and $\Gamma-z$ distribution of type-II AGN with radio counterparts (Fig. 10) which leads us to propose that J123642+621331, at $z=4.424$ with observed $\Gamma=1.35_{-0.40}^{+0.34}$, also contains a type-II AGN. Twelve of the type-II AGN (including this source) have radio starburst hosts.

The great majority (22/27) of 15 or $16 \mu$ m detections among the radio+X-ray sources are not type-II AGN hosts. They have a mean redshift of 1 and a mean photon index of 1.6. They appear more analogous to local ULIRGS than to the more extreme SMGs. Well-studied ULIRGs such as Arp 220 or Markarian 231 possess nuclear starbursts which would be barely resolved at the maximum detectable $z \sim 1$. Bauer et al. (2004) noted that the relative number counts of starburst and AGN X-ray sources changed from a large AGN majority over most of the flux density range sampled by Chandra to a higher proportion of starbursts among the faintest sources, likely to correspond to the less active tail of a relatively nearby population. The faintest $\sim 40 \%$ of $\mathrm{X}$-ray sources which are radio-quiet, but have a significant radio flux density revealed by stacking, (Sect. 8.2), may also be ULIRGs.

In contrast, the SMG counterparts have a mean redshift of 1.8 and an average photon index of 0.6 , suggesting that type-II AGN contribute substantially more of the X-ray emission. Alexander et al. (2005b) suggest that between a third and a half of the entire SCUBA source population contain AGN. 2/3 of radio-bright sources with X-ray and SCUBA emission contain type-II AGN. About $40 \%$ of the radio-faint sources found by stacking are associated with hard X-ray sources are probably also radio starbursts hosting obscured AGN. The radio starbursts appear to be extended over $2-10 \mathrm{kpc}$ or more, an order of magnitude larger than those in the local universe. The SMG (at a median redshift of at least 2.2) have star formation rates of the order of $1000 M_{\odot} \mathrm{yr}^{-1}$, also about 10 times higher than that of local ULIRGS.

\subsection{Implications for galaxy evolution}

Both starburst activity and feeding a black hole probably result from major mergers, which are increasingly common at $z>1.5$ and dominate galaxy growth at $z>2$ (Conselice et al. 2005). The comoving luminosity densities for AGN and for starbursts increases as $(1+z)^{\gtrless 3}$ for $z \lesssim 1.5$, using optical, IR, X-ray and radio classifications and luminosities (Barger et al. 2005; Cowie et al. 2004a). Current estimates of the star formation rate as a function of redshift (Hopkins \& Beacom 2006) suggest that the star formation rate continues to increase out to redshifts of 3 or more. It is possible that sub-mm galaxies at unknown redshifts may be even more vigorous starbursts at $z \gtrsim 3$ (Ivison et al. 2007).

The one radio-bright object in the $\operatorname{HDF}(\mathrm{N})$ with a known redshift greater than $3, \mathrm{~J} 123642+621331$ at $z=4.424$, appears to contain both a (probably obscured) AGN and a very productive starburst. Stacking $1.4-\mathrm{GHz}$ emission from radio-faint sources at the position of objects detected by Chandra shows a statistically significant excess which rises with decreasing $\Gamma$ and with increasing hard-band X-ray flux densities (Sect. 8.2). Eleven individual radio-faint counterparts to X-ray sources are $>5 \sigma(>20 \mu \mathrm{Jy})$, all of which appear extended and lacking radio hotspots, consistent with these sources being a faint or highredshift tail of the radio starbursts associated with type-II AGN.

Within the next 2 years, $e$-MERLIN+EVLA images should reveal many more high-redshift $\mu \mathrm{Jy}$ galaxies with optical, Spitzer or SCUBA(2) counterparts, pushing measurements of the star formation rate (and redshifts) back to $z \gtrsim 5$, whilst the ongoing increase in capacity of the VLBI correlator at JIVE will enable distant compact radio AGN cores to be distinguished from even sub-kpc-scale starbursts (Garrett et al. 2005).

Acknowledgements. This work is based in part on observations made with the Multi Element Radio-Linked Interferometer Network (MERLIN), operated by Manchester University on behalf of PPARC, and with the Very Large Array, a facility of the NSF operated under cooperative agreement by Associated Universities, Inc. This work has benefited from research funding from the EC Framework 6 Programme under RadioNet R113CT 2003 5058187. We employed software provided by the UK AstroGrid Virtual Observatory Project, funded by PPARC and the Framework 6 Programme and we also used the VizieR catalogue access tool and the ADS bibliographic service.

We thank the MERLIN and VLA staff for considerable assistance with observations. A.M.S.R. acknowledges the hospitality provided by CDS, where she was a "professeur invité" during part of this work. We thank P. Padovani (ESO) for helpful discussions and we are extremely grateful to the anonymous referee for improving the accuracy, clarity and consistency of the paper.

\section{References}

Alexander, D. M., Aussel, H., Bauer, F. E., et al. 2002, ApJ, 568, L85 Alexander, D. M., Bauer, F. E., Brandt, W. N., et al. 2003a, AJ, 125, 383 Alexander, D. M., Bauer, F. E., Brandt, W. N., et al. 2003b, AJ, 126, 539 Alexander, D. M., Bauer, F. E., Chapman, S. C., et al. 2005a, ApJ, 632, 736 Alexander, D. M., Smail, I., Bauer, F. E., et al. 2005b, Nature, 434, 738 Aussel, H., Cesarsky, C. J., Elbaz, D., \& Starck, J. L. 1999, A\&A, 342, 313 Barger, A. J., Cowie, L. L., Trentham, N., et al. 1999, AJ, 117, 102 Barger, A. J., Cowie, L. L., Brandt, W. N., et al. 2002, AJ, 124, 1839 Barger, A. J., Cowie, L. L., Capak, P., et al. 2003, AJ, 126, 632 Barger, A. J., Cowie, L. L., Mushotzky, R. F., et al. 2005, AJ, 129, 578 Barger, A. J., Cowie, L. L., \& Wang, W.-H. 2007, ApJ, 654, 764 Barthel, P. D., \& Miley, G. K. 1988, Nature, 333, 319

Bauer, F. E., Alexander, D. M., Brandt, W. N., et al. 2002a, AJ, 123, 1163 Bauer, F. E., Alexander, D. M., Brandt, W. N., et al. 2002b, AJ, 124, 2351 Bauer, F. E., Alexander, D. M., Brandt, W. N., et al. 2004, AJ, 128, 2048 Beswick, R. J., Muxlow, T. W. B., Thrall, H., \& Richards, A. M. S. 2006, in At the Edge of the Universe, ed. J. Alfonso, H. Ferguson, \& R. Norris, ASP Conf. Ser. [arXiv: astro-ph/0612077]

Blain, A. W., Smail, I., Ivison, R. J., Kneib, J.-P., \& Frayer, D. T. 2002, Phys. Rep., 369, 111

Borys, C., Scott, D., Chapman, S., et al. 2004, MNRAS, 355, 485

Chapman, S. C., Smail, I., Blain, A. W., \& Ivison, R. J. 2004a, ApJ, 614, 671

Chapman, S. C., Smail, I., Windhorst, R., Muxlow, T., \& Ivison, R. J. 2004b, ApJ, 611, 732

Chapman, S. C., Blain, A. W., Smail, I., \& Ivison, R. J. 2005, ApJ, 622, 772

Chi, S., Garrett, M. A., \& Barthel, P. D. 2006, in 8th EVN Symposium, ed. A. Marecki, Proceedings of Science (SISSA, Trieste),

http://os.sissa.it/archive/conferences/036/

Cohen, J. G. 2001, AJ, 121, 2895

Cohen, J. G., Cowie, L. L., Hogg, D. W., et al. 1996, ApJ, 471, L5

Cohen, J. G., Hogg, D. W., Blandford, R., et al. 2000, ApJ, 538, 29

Condon, J. J. 1992, ARA\&A, 30, 575

Conselice, C. J., Bershady, M. A., Dickinson, M., \& Papovich, C. 2003, AJ, 126, 1183

Conselice, C. J., Blackburne, J. A., \& Papovich, C. 2005, ApJ, 620, 564 
Cowie, L. L., Barger, A. J., Fomalont, E. B., \& Capak, P. 2004a, ApJ, 603, L69 Cowie, L. L., Barger, A. J., Hu, E. M., Capak, P., \& Songaila, A. 2004b, AJ, 127, 3137

Cram, L., Hopkins, A., Mobasher, B., \& Rowan-Robinson, M. 1998, ApJ, 507, 155

Daddi, E., Cimatti, A., Renzini, A., et al. 2004, ApJ, 600, L127

Daddi, E., Dickinson, M., Chary, R., et al. 2005, ApJ, 631, L13

Dawson, S., Stern, D., Bunker, A. J., Spinrad, H., \& Dey, A. 2001, AJ, 122, 598

Downes, D., \& Solomon, P. M. 1998, ApJ, 507, 615

Elbaz, D., Cesarsky, C. J., Chanial, P., et al. 2002, A\&A, 384, 848

Evans, D. A., Worrall, D. M., Hardcastle, M. J., Kraft, R. P., \& Birkinshaw, M. 2006, ApJ, 642, 96

Fanaroff, B. L., \& Riley, J. M. 1974, MNRAS, 167, 31P

Fomalont, E. B., Kellermann, K. I., \& Partridge, R. B., et al. 2002, AJ, 123, 2402

Frayer, D. T., Ivison, R. J., Scoville, N. Z., et al. 1998, ApJ, 506, L7

Garrett, M. A. 2002, A\&A, 384, L19

Garrett, M. A., de Bruyn, A. G., Giroletti, M., Baan, W. A., \& Schilizzi, R. T. 2000, A\&A, 361, L41

Garrett, M. A., Muxlow, T. W. B., Garrington, S. T., et al. 2001, A\&A, 366, L5

Garrett, M. A., Wrobel, J. M., \& Morganti, R. 2005, in Future Directions in High Resolution Astronomy, ed. J. Romney, \& M. Reid, ASP Conf. Ser., 340, 600 George, I. M., Turner, T. J., Yaqoob, T., et al. 2000, ApJ, 531, 52

Giavalisco, M., Ferguson, H. C., Koekemoer, A. M., et al. 2004, ApJ, 600, L93

Goldschmidt, P., Oliver, S. J., Serjeant, S. B. G., et al. 1997, MNRAS, 289, 465

Griffiths, R. E., Ptak, A., Feigelson, E. D., et al. 2000, Science, 290, 1325

Grimm, H.-J., Gilfanov, M., \& Sunyaev, R. 2003, MNRAS, 339, 793

Hopkins, A. M., \& Beacom, J. F. 2006, ApJ, 651, 142

Hornschemeier, A. E., Brandt, W. N., Garmire, G. P., et al. 2001, ApJ, 554, 742

Hughes, D. H., Serjeant, S., Dunlop, J., et al. 1998, Nature, 394, 241

Ivison, R. J., Chapman, S. C., Faber, S. M., et al. 2007, ApJ, 660, L77

Kettenis, M. M., van Langevelde, H. J., Reynolds, C., \& Cotton, B. 2006, in Astronomical Data Analysis Software and Systems XV, ed. C. Gabriel, C. Arviset, D. Ponz, \& S. Enrique, ASP Conf. Ser., 351, 497
Madau, P., Ferguson, H. C., Dickinson, M. E., et al. 1996, MNRAS, 283, 1388 Morrison, G., Dickinson, M., Owen, F., et al. 2006, in ASP Conf. Ser. Infrared Diagnostics of Galaxy Evolution (Spitzer Conference), ed. R.-R. Chary [arXiv:astro-ph/0603412]

Muxlow, T. W. B., Richards, A. M. S., Garrington, S. T., et al. 2005, MNRAS, 358,1159

Muxlow, T. W. B., Beswick, R. J., Thrall, H., et al. 2006, in At the Edge of the Universe, ed. J. Alfonso, H. Ferguson, \& R. Norris, ASP Conf. Ser.

Norman, C., Ptak, A., Hornschemeier, A., et al. 2004, ApJ, 607, 721

Padovani, P., Allen, M. G., Rosati, P., \& Walton, N. A. 2004, A\&A, 424, 545

Pope, A., Borys, C., Scott, D., et al. 2005, MNRAS, 358, 149

Ptak, A., Serlemitsos, P., Yaqoob, T., \& Mushotzky, R. 1999, ApJS, 120, 179

Ranalli, P., Comastri, A., \& Setti, G. 2003, A\&A, 399, 39

Reddy, N. A., \& Steidel, C. C. 2004, ApJ, 603, L13

Richards, E. A. 2000, ApJ, 533, 611

Richards, E. A., Kellermann, K. I., Fomalont, E. B., Windhorst, R. A., \& Partridge, R. B. 1998, AJ, 116, 1039

Rosa-Gonzalez, D., Burgarella, D., Nandra, K., et al. 2007, MNRAS, [arXiv:0705.1278v1]

Sadler, E. M., Jackson, C. A., Cannon, R. D., et al. 2002, MNRAS, 329, 227

Serjeant, S., Dunlop, J. S., Mann, R. G., et al. 2003, MNRAS, 344, 887 Smail, I., Ivison, R. J., Blain, A. W., \& Kneib, J.-P. 2002, MNRAS, 331, 495

Snellen, I. A. G., \& Best, P. N. 2001, MNRAS, 328, 897

Soifer, B. T., Neugebauer, G., Matthews, K., et al. 2000, AJ, 119, 509

Szokoly, G. P., Bergeron, J., Hasinger, G., et al. 2004, ApJS, 155, 271

Teplitz, H. I., Charmandaris, V., Chary, R., et al. 2005, ApJ, 634, 128

Waddington, I., Windhorst, R. A., \& Cohen, S. H., et al. 1999, ApJ, L77

Wang, W.-H., Cowie, L. L., \& Barger, A. J. 2004, ApJ, 613, 655

Williams, R. E., Blacker, B., Dickinson, M., et al. 1996, AJ, 112, 1335

Yun, M. S., \& Carilli, C. L. 2002, ApJ, 568, 88

Yun, M. S., Reddy, N. A., \& Condon, J. J. 2001, ApJ, 554, 803

Zirm, A. W., van der Wel, A., Franx, M., et al. 2007, ApJ, 656, 66 